\title{
Discovery of a directly imaged planet to the young solar analog YSES $2^{\star, \star \star}$
}

\author{
Alexander J. Bohn ${ }^{1}$, Christian Ginski ${ }^{2,1}$, Matthew A. Kenworthy ${ }^{1}$, Eric E. Mamajek ${ }^{3,4}$, Mark J. Pecaut ${ }^{5}$, \\ Markus Mugrauer ${ }^{6}$, Nikolaus Vogt ${ }^{7}$, Christian Adam ${ }^{7,8}$, Tiffany Meshkat ${ }^{9}$, Maddalena Reggiani ${ }^{10}$, and Frans Snik $^{1}$ \\ ${ }^{1}$ Leiden Observatory, Leiden University, PO Box 9513, 2300 RA Leiden, The Netherlands \\ e-mail: bohn@strw. leidenuniv.nl \\ 2 Sterrenkundig Instituut Anton Pannekoek, Science Park 904, 1098 XH Amsterdam, The Netherlands \\ 3 Jet Propulsion Laboratory, California Institute of Technology, 4800 Oak Grove Drive, M/S 321-100, Pasadena, CA 91109, USA \\ ${ }^{4}$ Department of Physics \& Astronomy, University of Rochester, Rochester, NY 14627, USA \\ 5 Rockhurst University, Department of Physics, 1100 Rockhurst Road, Kansas City, MO 64110, USA \\ ${ }^{6}$ Astrophysikalisches Institut und Universitäts-Sternwarte Jena, Schillergäßchen 2, 07745 Jena, Germany \\ ${ }^{7}$ Instituto de Física y Astronomía, Facultad de Ciencias, Universidad de Valparaíso, Av. Gran Bretaña 1111, Playa Ancha, Valparaíso, \\ Chile \\ ${ }^{8}$ Núcleo Milenio Formación Planetaria - NPF, Universidad de Valparaíso, Av. Gran Bretaña 1111, Valparaíso, Chile \\ 9 IPAC, California Institute of Technology, M/C 100-22, 1200 East California Boulevard, Pasadena, CA 91125, USA \\ ${ }^{10}$ Institute of Astronomy, KU Leuven, Celestijnenlaan 200D, 3001 Leuven, Belgium
}

Received 6 February 2021 / Accepted 17 March 2021

\begin{abstract}
Context. To understand the origin and formation pathway of wide-orbit gas giant planets, it is necessary to expand the limited sample of these objects. The mass of exoplanets derived with spectrophotometry, however, varies strongly as a function of the age of the system and the mass of the primary star.

Aims. By selecting stars with similar ages and masses, the Young Suns Exoplanet Survey (YSES) aims to detect and characterize planetary-mass companions to solar-type host stars in the Scorpius-Centaurus association.

Methods. Our survey is carried out with VLT/SPHERE with short exposure sequences on the order of 5 min per star per filter. The subtraction of the stellar point spread function (PSF) is based on reference star differential imaging using the other targets (with similar colors and magnitudes) in the survey in combination with principal component analysis. Two astrometric epochs that are separated by more than one year are used to confirm co-moving companions by proper motion analysis.

Results. We report the discovery of YSES 2b, a co-moving, planetary-mass companion to the K1 star YSES 2 (TYC 8984-2245-1, 2MASS J11275535-6626046). The primary has a Gaia EDR3 distance of $110 \mathrm{pc}$, and we derive a revised mass of $1.1 M_{\odot}$ and an age of approximately $14 \mathrm{Myr}$. We detect the companion in two observing epochs southwest of the star at a position angle of $205^{\circ}$ and with a separation of $\sim 1^{\prime \prime} 05$, which translates to a minimum physical separation of 115 au at the distance of the system. Photometric measurements in the $H$ and $K_{\mathrm{s}}$ bands are indicative of a late $L$ spectral type, similar to the innermost planets around HR 8799 . We derive a photometric planet mass of $6.3_{-0.9}^{+1.6} M_{\text {Jup }}$ using AMES-COND and AMES-dusty evolutionary models; this mass corresponds to a mass ratio of $q=(0.5 \pm 0.1) \%$ with the primary. This is the lowest mass ratio of a direct imaging planet around a solar-type star to date. We discuss potential formation mechanisms and find that the current position of the planet is compatible with formation by disk gravitational instability, but its mass is lower than expected from numerical simulations. Formation via core accretion must have occurred closer to the star, yet we do not find evidence that supports the required outward migration, such as via scattering off another undiscovered companion in the system. We can exclude additional companions with masses greater than $13 M_{\text {Jup }}$ in the full field of

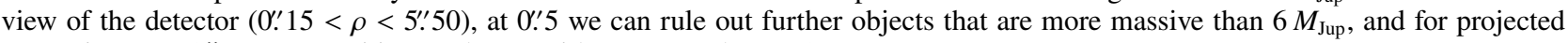
separations $\rho>2^{\prime \prime}$ we are sensitive to planets with masses as low as $2 M_{\text {Jup. }}$.

Conclusions. YSES $2 \mathrm{~b}$ is an ideal target for follow-up observations to further the understanding of the physical and chemical formation mechanisms of wide-orbit Jovian planets. The YSES strategy of short snapshot observations $(\leq 5 \mathrm{~min})$ and PSF subtraction based on a large reference library proves to be extremely efficient and should be considered for future direct imaging surveys.
\end{abstract}

Key words. planets and satellites: detection - planets and satellites: formation - instrumentation: high angular resolution techniques: image processing - stars: individual: TYC 8984-2245-1

\footnotetext{
${ }^{\star}$ Data are only available at the CDS via anonymous ftp to cdsarc.u-strasbg.fr (130.79.128.5) or via http://cdsarc. u-strasbg.fr/viz-bin/cat/J/A+A/648/A73

$\star \star$ Based on observations collected at the European Organisation for Astronomical Research in the Southern Hemisphere under ESO programs 099.C-0698(A), 0101.C-0153(A), 0101.C-0341(A), and 106.20X2.001.
}

\section{Introduction}

Despite several remarkable exoplanet and brown dwarf discoveries by high-contrast imaging at high angular resolution in the past few years (e.g., Marois et al. 2008, 2010; Schmidt et al. 2008; Lagrange et al. 2010; Rameau et al. 2013; Bailey et al. 2014; Macintosh et al. 2015; Chauvin et al. 2017; Keppler et al. 2018; Haffert et al. 2019; Janson et al. 2019; Bohn et al. 2020a,b), 
there is an ongoing debate regarding the formation mechanisms that create these super-Jovian gas giants with semimajor axes greater than $10 \mathrm{au}$. It is unclear whether these companions have a star-like origin from a collapsing molecular cloud that is broken up into fragments, creating planetary-mass objects similar to a stellar binary (Kroupa 2001; Chabrier 2003), or through formation in a circumstellar disk instead. The classical bottom-up framework postulates formation via core accretion by coagulation of small dust grains into planetary embryos (Pollack et al. 1996; Alibert et al. 2005; Dodson-Robinson et al. 2009; Lambrechts \& Johansen 2012). These evolve either via collisions or pebble accretion (Johansen \& Lacerda 2010; Ormel \& Klahr 2010) into planetary cores that are massive enough to accrete a gaseous envelope and to open a gap in the disk (Paardekooper \& Mellema 2004). In the corresponding top-down scenario, planetary cores can be created by gravitational instabilities leading to the collapse of dense regions in the protoplanetary disk (Boss 1997, 2011; Rafikov 2005; Durisen et al. 2007; Kratter et al. 2010; Kratter \& Lodato 2016).

To study this question for the underlying planet formation mechanisms from a statistical point of view, several direct imaging surveys have been conducted (e.g., Vigan et al. 2012, 2017; Galicher et al. 2016; Bowler 2016). Synthetic planet populations that represent each of the potential formation channels can be compared to the observational results from surveys and place constraints on the efficiency of the corresponding formation pathway (e.g., Mordasini et al. 2009a,b; Forgan \& Rice 2013; Forgan et al. 2018). The two largest surveys were carried out with two of the most advanced adaptive-optics assisted, high-contrast imagers available: the Spectro-Polarimetric High-contrast Exoplanet REsearch (SPHERE; Beuzit et al. 2019) instrument at the $8.2 \mathrm{~m} \mathrm{ESO/VLT}$ and the Gemini Planet Imager (GPI; Macintosh et al. 2014) at the $8.1 \mathrm{~m}$ Gemini South telescope. The preliminary statistical analysis of the first 300 stars from the Gemini PLanet Imager Exoplanet Survey (GPIES; Nielsen et al. 2019) concludes that giant planets between $10 \mathrm{au}$ and $100 \mathrm{au}$ that have masses smaller than $13 M_{\text {Jup }}$ favorably form via core accretion mechanisms, whereas brown dwarf companions in the same separation range but with masses from $13 M_{\text {Jup }}$ to $80 M_{\text {Jup }}$ seem to be predominantly created by disk instabilities. This finding is supported by the analysis of the first 150 stars observed within the scope of the SpHere INfrared survey for Exoplanets (SHINE; Vigan et al. 2021), which additionally hypothesizes that companions with masses between $1 M_{\text {Jup }}$ and $75 M_{\text {Jup }}$ are likely to originate from bottom-up formation scenarios around B and A type stars, whilst objects of the same mass around M-type stars are consistent with simulated populations from top-down mechanisms. For the intermediate masses of F-, G-, and K-type stars, the observed detections can be explained by a combination of both formalisms. A statistical meta-analysis on the distribution of wide-orbit companion eccentricities carried out by Bowler et al. (2020) provides supporting evidence for two distinct formation channels shaping the populations of giant planets $\left(2 M_{\text {Jup }}<M<15 M_{\text {Jup }}\right)$ and brown dwarfs $\left(15 M_{\text {Jup }}<\right.$ $\left.M<75 M_{\text {Jup }}\right)$.

Most of these statistical evaluations are affected by the small number of actual substellar companions that were detected in the preceding imaging surveys. To expand the sample size for solar-mass host stars, we started the Young Suns Exoplanet Survey (YSES; Bohn et al. 2020a, and in prep.) that is observing a homogeneous sample of $70 \sim 15$ Myr-old, K-type stars in the Lower Centaurus Crux (LCC) subgroup of the ScorpiusCentaurus association (Sco-Cen; de Zeeuw et al. 1999). All stars have masses close to $1 M_{\odot}$ and the proximity (average parallactic distance $\langle D\rangle=114 \pm 17$; Gaia Collaboration 2021) and youth of the LCC facilitate the direct imaging search of giant, self-luminous substellar companions around these stars.

In this article, we report the detection of a new exoplanet that was discovered within the scope of our survey. As this is already the second planetary system discovered by YSES, we introduce a new stellar identifier that is based on our survey acronym. The details of this new designation are described in Sect. 2. In Sect. 3, we describe our observations and data reduction methods. We discuss previous observations on the host star and reassess its main parameters in Sect. 4. The results of our high-contrast imaging observations are presented and analyzed in Sect. 5. We discuss potential formation mechanisms of this newly detected exoplanet in Sect. 6 and we present our conclusions in Sect. 7.

\section{Nomenclature of YSES planets}

Owing to the recent success of YSES, we decided to introduce a dedicated catalog that will be used for star-planet systems discovered within the scope of our survey. The YSES acronym has been verified by the IAU Commission B2 Working Group on Designations and was added to the Simbad database (Wenger et al. 2000) ${ }^{1}$. The nomenclature of planet hosts from our survey is YSES NNN and planets that are associated with these stars will be named YSES NNNa, accordingly. Following these guidelines, we assigned the host star of the intriguing multi-planet system that was discovered around TYC 8998-760-1 the new primary identifier YSES 1 (Bohn et al. 2020a,b). The planets formerly known as TYC 8998-760-1 b and TYC 8998-760-1 c, will be named YSES $1 \mathrm{~b}$ and YSES 1c, henceforth. Further planetary systems discovered by our survey will receive designated YSES identifiers followed by ascending integer identifications (IDs). Hence, the new companion discovered within the scope of this paper will be referred to as YSES 2b, orbiting its Sun-like host YSES 2.

\section{Observations and data reduction}

We observed YSES 2 (TYC 8984-2245-1, 2MASS J112755356626046) as part of YSES on the nights of 2018 April 30 (PI: Kenworthy) and 2020 December 8 (PI: Vogt) with SPHERE (mounted at the Nasmyth platform of Unit Telescope 3 of the ESO Very Large Telescope). We used the IRDIS camera (Dohlen et al. 2008) in classical imaging mode, applying a broadband filter in the $H$ and $K_{\mathrm{s}}$ bands during the first and second nights, respectively. The observations were carried out in pupil stabilized imaging mode and an apodized Lyot coronagraph was used to block the flux of the primary star (Soummer 2005; Martinez et al. 2009; Carbillet et al. 2011). In addition to the science frames, we obtained center frames, with a sinusoidal pattern applied to the deformable mirror that creates a waffle pattern to locate the position of the star behind the coronagraphic mask; sky frames of an offset position with no adaptive optics (AO) correction and without any source in the field of view, to subtract the instrument and thermal background; and non-coronagraphic images of the star that are used for photometric reference of point sources detected in the science images. This last category of non-coronagraphic flux images was obtained with an additional neutral density filter in the optical path to record an

1 Database entry available at: http://cds.u-strasbg.fr/ cgi-bin/Dic-Simbad?/18721212 
unsaturated stellar point spread function (PSF) in the linear readout regime of the detector. This neutral density filter (filter ID: ND_1.0) provided an attenuation of 7.9 and 6.9 across the $H$ and $K_{\mathrm{s}}$ bandpasses, respectively. A detailed description of the observing setup and the weather conditions can be found in Appendix A.

The data reduction was performed with PynPoint (version 0.8.1; Stolker et al. 2019) and included basic processing steps such as dark and flat calibration, bad pixel cleaning, sky subtraction, and correction for the instrumental distortion along the vertical axis of the detector. To remove the stellar halo that is affecting approximately the innermost 1.2 around the coronagraph, we utilized an approach based on reference star differential imaging (RDI; Smith \& Terrile 1984) in combination with principal component analysis (PCA; Amara \& Quanz 2012; Soummer et al. 2012). As the parallactic rotation of our YSES observations is usually less than a few degrees, classical PSF subtraction schemes, such as angular differential imaging (ADI; Marois et al. 2006), perform much worse compared to this reference library approach. This method of combined RDI plus PCA was already successfully employed to recover circumstellar disks in archival HST data (e.g., Choquet et al. 2014) and within the scope of our survey for the discovery of a transition disk around the YSES target Wray 15-788 (Bohn et al. 2019).

Owing to the same location on sky, similar distances, and spectral types, all YSES targets exhibit very similar magnitudes in the red part of the optical spectrum (where the wavefront sensor of SPHERE is operating) and at the near-infrared wavelengths of our scientific observations. This facilitates comparable AO corrections amongst all our YSES observations, and the resulting images compose an excellent reference library to perform RDI. The reference targets that were used for our library PSF subtraction are listed in Appendix B. We modeled the stellar PSF with 50 principal components that were obtained from our full reference library. After the PSF subtraction, the frames were de-rotated according to their parallactic angles and median combined.

For the astrometric calibration we used the standard instrumental solution as presented by Maire et al. (2016) with a wavelength independent true north offset of $-1.75 \pm 0.08$ and plate scales of $(12.251 \pm 0.010)$ mas px $^{-1}$ and $(12.265 \pm$ $0.010)$ mas px $^{-1}$ in the $H$ and $K_{\mathrm{s}}$ band, respectively.

\section{Stellar properties}

We briefly summarize previous literature characterizing YSES 2 in Sect. 4.1 and compile the stellar properties of YSES 2 in Table 1. In Sects. 4.2 and 4.3, we derive updated stellar parameters for YSES 2, more importantly, including stellar mass and age.

\subsection{Previous studies}

YSES 2 was first identified as a young star in the Search for Associations Containing Young stars (SACY) survey (Torres et al. 2006) of optical counterparts to the ROSAT All-Sky Survey (RASS) X-ray sources (Voges et al. 1999). Torres et al. (2006) reported the star to be a Li-rich $(\mathrm{EW}[\mathrm{Li}$ I 16707] $=367 \mathrm{m \AA})$ $\mathrm{K} 1 \mathrm{~V}$ (e) star with filled-in $\mathrm{H} \alpha$, showing fast rotation $(v \sin i=$ $19.3 \mathrm{~km} \mathrm{~s}^{-1}$ ) and radial velocity $15.8 \pm 1.0 \mathrm{~km} \mathrm{~s}^{-1}$. Based on its position, proper motion, and youth indicators, Preibisch \& Mamajek (2008) included the star in a list of new members of the LCC subgroup of the Sco-Cen OB association (their Table 4), and provided initial estimates of isochronal age (16 Myr), mass
Table 1. Stellar properties of YSES 2.

\begin{tabular}{|c|c|c|}
\hline Parameter & Value & Ref. \\
\hline Main identifier & YSES 2 & (1) \\
\hline Tycho ID & TYC 8984-2245-1 & (2) \\
\hline 2MASS ID & J11275535-6626046 & (3) \\
\hline Gaia EDR3 ID & 5236792880333011968 & (4) \\
\hline$\alpha(\mathrm{J} 2000)(\mathrm{hh}$ mm ss.sss $)$ & 112755.355 & (4) \\
\hline$\delta(\mathrm{J} 2000)(\mathrm{dd}$ mm ss.ss $)$ & -662604.50 & (4) \\
\hline Spectral Type & $\mathrm{K} 1 \mathrm{~V}(\mathrm{e})$ & $(5)$ \\
\hline$\varpi$ (mas) & $9.1537 \pm 0.0118$ & (4) \\
\hline$D(\mathrm{pc})$ & $109.25 \pm 0.14$ & (7) \\
\hline$\mu_{\alpha *}\left(\operatorname{mas~yr}^{-1}\right)$ & $-34.025 \pm 0.013$ & (4) \\
\hline$\mu_{\delta}\left(\operatorname{mas} \mathrm{yr}^{-1}\right)$ & $2.319 \pm 0.011$ & (4) \\
\hline$v_{\text {rad }}\left(\mathrm{km} \mathrm{s}^{-1}\right)$ & $13.41 \pm 0.17$ & (5) \\
\hline$B(\mathrm{mag})$ & $11.819 \pm 0.010$ & (8) \\
\hline$V(\mathrm{mag})$ & $10.860 \pm 0.017$ & $(8)$ \\
\hline$G(\mathrm{mag})$ & $10.525 \pm 0.003$ & (4) \\
\hline$I$ (mag) & $9.773 \pm 0.044$ & (9) \\
\hline$J(\mathrm{mag})$ & $9.006 \pm 0.026$ & (3) \\
\hline$H$ (mag) & $8.484 \pm 0.029$ & (3) \\
\hline$K_{\mathrm{s}}(\mathrm{mag})$ & $8.358 \pm 0.029$ & (3) \\
\hline$W 1$ (mag) & $8.323 \pm 0.014$ & (10) \\
\hline$W 2$ (mag) & $8.351 \pm 0.008$ & $(10)$ \\
\hline W3 (mag) & $8.258 \pm 0.019$ & (11) \\
\hline W4 (mag) & $7.929 \pm 0.118$ & (11) \\
\hline$P_{\text {rot }}$ (day) & 2.7325 & (9) \\
\hline$v \sin i\left(\mathrm{~km} \mathrm{~s}^{-1}\right)$ & $19.3 \pm 0.5$ & (5) \\
\hline $\log \left(L_{\mathrm{X}} / L_{\mathrm{bol}}\right)(\mathrm{dex})$ & $-3.07 \pm 0.23$ & (9) \\
\hline EW(Li I 16707$)(\mathrm{mA})$ & $364 \pm 0.05$ & (6) \\
\hline $\mathrm{EW}(\mathrm{H} \alpha)(\mathrm{m \AA})$ & 0.0 & $(5)$ \\
\hline$U\left(\mathrm{~km} \mathrm{~s}^{-1}\right)$ & $-10.10 \pm 0.08$ & (7) \\
\hline$V\left(\mathrm{~km} \mathrm{~s}^{-1}\right)$ & $-18.93 \pm 0.12$ & (7) \\
\hline$W\left(\mathrm{~km} \mathrm{~s}^{-1}\right)$ & $-5.60 \pm 0.09$ & (7) \\
\hline$A_{\mathrm{V}}(\mathrm{mag})$ & $0.06_{-0.04}^{+0.03}$ & (6) \\
\hline$T_{\text {eff }}(\mathrm{K})$ & $4749 \pm 40$ & (6) \\
\hline$m_{\text {bol }}(\mathrm{mag})$ & $10.396 \pm 0.015$ & (6) \\
\hline$M_{\mathrm{bol}}(\mathrm{mag})$ & $5.204 \pm 0.016$ & (6) \\
\hline $\log \left(L / L_{\odot}\right)(\operatorname{dex})$ & $-0.1854 \pm 0.0063$ & (6) \\
\hline$R\left(R_{\odot}\right)$ & $1.193 \pm 0.022$ & (6) \\
\hline $\operatorname{Mass}\left(M_{\odot}\right)$ & $1.10 \pm 0.03$ & (6) \\
\hline Age (Myr) & $13.9 \pm 2.3$ & (6) \\
\hline
\end{tabular}

References. (1) This paper, see Sect. 2; (2) Høg et al. (2000); (3) Cutri et al. (2003); (4) Gaia Collaboration (2021), and Gaia EDR3 and DR2 ID \#s are the same; (5) Torres et al. (2006); (6) this paper, see Sect. 4.3; (7) distance and heliocentric Galactic Cartesian velocity calculated using Gaia EDR3 values ( $D=1 / \varpi)$; (8) Henden et al. (2016); (9) Kiraga (2012); (10) Eisenhardt et al. (2020); (11) Cutri \& et al. (2014).

$\left(1.1 M_{\odot}\right)$, and fractional X-ray luminosity $\left(\log \left(L_{\mathrm{X}} / L_{\mathrm{bol}}\right)=-3.2\right)$. Preibisch \& Mamajek (2008) also predicted a kinematic distance of $109 \mathrm{pc}$ (based on the proper motion and space velocity of LCC), which compares remarkably well to the Gaia EDR3 parallactic distance $(\varpi=9.1537 \pm 0.0118$ mas, $D=109.25 \pm 0.14$ pc; Gaia Collaboration 2021). Kiraga (2012) reported the star to be a variable in the All Sky Automatic Survey (ASAS; IDed as ASAS J112755-6625.9) showing high amplitude $(0.093 \mathrm{mag}$ in $\mathrm{V})$ and rapid rotation $\left(P_{\text {rot }}=2.7325 \mathrm{~d}\right)$, which is consistent with the observed saturated X-ray emission (and right near the median rotation period for Sco-Cen premain-sequence stars of $\left\langle P_{\text {rot }}\right\rangle \simeq 2.4$ days; Mellon et al. 2017). 
Pecaut \& Mamajek (2016) include the star in their age analysis of pre-main-sequence $\mathrm{K}$ stars across Sco-Cen, estimating an age of $23 \mathrm{Myr}$ and a mass of $1.0 \mathrm{M}_{\odot}$. The star has subsequently appeared in multiple LCC membership lists (Gagné et al. 2018; Goldman et al. 2018; Damiani et al. 2019). The status of this star as a pre-main-sequence member of LCC is strongly corroborated by a $99.9 \%$ membership probability from the BANYAN $\Sigma$ algorithm (Gagné et al. 2018) applied to the available Gaia astrometry and radial velocities (Gaia Collaboration 2018, 2021).

\subsection{Spectral analysis of YSES 2}

To check the previously published spectral properties of YSES 2, we examined two archival UVES spectra from the ESO archive taken on UT 2007 May 2 (Program 079.C-0556(A); PI Torres). The UVES spectra at resolution $R=40000$ were convolved to lower resolution $R=3000$ and compared to the grid of MK spectral standards from Pecaut \& Mamajek (2016). The blue spectrum, 3280-4560 $\mathrm{A}$, is consistent with $\mathrm{K} 0 \mathrm{~V}$, but the red spectrum, 4730-6840 $\AA$, appears to be K2V. The H $\alpha$ line exhibits marginal emission, similar to the filled-in emission reported by Torres et al. (2006). Hence, we infer a temperature type of $\mathrm{K} 1 \pm 1$ and confirm the type $\mathrm{K} 1 \mathrm{~V}(\mathrm{e})$ published by Torres et al. (2006). From the original spectra, we independently measure the equivalent width of the Li I $\lambda 6707$ feature to be $364 \pm 5 \mathrm{~m} \AA$, by simultaneously fitting Voigt profiles to the Li I feature and Fe I blend nearby (see, e.g., Soderblom et al. 1993). This is in good agreement with the $367 \mathrm{~m} \AA$ reported by Torres et al. (2006). The assigned luminosity class that we adapted from Torres et al. (2006) does not necessarily imply that YSES 2 is on the main sequence rather than being a pre-main-sequence star. Even though the luminosity and gravity indicators used by Torres et al. (2006) were more in line with main-sequence dwarfs than subgiant or giant standard stars, more persuasive indicators such as the HRD position, Li absorption, X-ray emission, and the confirmed LCC membership clearly favor the pre-main-sequence evolutionary stage.

\subsection{Updated stellar parameters}

Using the VOSA spectral energy distribution (SED) analyzer (Bayo et al. 2008) ${ }^{2}$, we fit synthetic stellar spectra to the observed visible and infrared photometry for YSES 2. For priors, we constrained the reddening to be $E(B-V)=$ $0.016 \pm 0.017 \mathrm{mag}$ based on the STILISM 3D reddening maps (Lallement et al. 2019) and searched for best-fit synthetic spectra in the range $3000 \mathrm{~K}<T_{\text {eff }}<6000 \mathrm{~K}, 3.5 \mathrm{dex}<\log g<4.5 \mathrm{dex}$, and metallicities $-0.5<[\mathrm{Fe} / \mathrm{H}]<0.5$ and $[\alpha / \mathrm{Fe}]=0.0$. A Bayesian fit using the BT-Settl-CIFIST models using 22 photometric points yielded the following parameters: $A_{\mathrm{V}}=0.06$ $(0.02-0.09 ; 68 \% \mathrm{CL} ; 0.00-0.11 ; 95 \% \mathrm{CL}), T_{\text {eff }}=4749 \mathrm{~K}(4709$ $4789 \mathrm{~K} ; 68 \% \mathrm{CL} ; 4700-4900 \mathrm{~K} ; 95 \% \mathrm{CL}), \log g=3.9 \operatorname{dex}(3.5-$ $4.5 \mathrm{dex}),[\mathrm{Fe} / \mathrm{H}]=0.0$. We present the results of this SED fit in Fig. 1 .

The best-fit bolometric flux is $f_{\text {bol }}=(1.7490 \pm 0.0248) \times$ $10^{-9} \mathrm{erg} \mathrm{s}^{-1} \mathrm{~cm}^{-2}$, which on the IAU 2015 apparent bolometric magnitude scale translates to $m_{\text {bol }}=10.396 \pm$ $0.015 \mathrm{mag}$. Adopting the Gaia EDR3 parallax, this translates to absolute bolometric magnitude $M_{\mathrm{Bol}}=5.204 \pm 0.016 \mathrm{mag}$ and bolometric luminosity $\log \left(L / L_{\odot}\right)=-0.1854 \pm 0.0063$. This is considerably more accurate than previous estimates

\footnotetext{
2 Online available at: http://svo2.cab.inta-csic.es/theory/ vosa/index.php
}

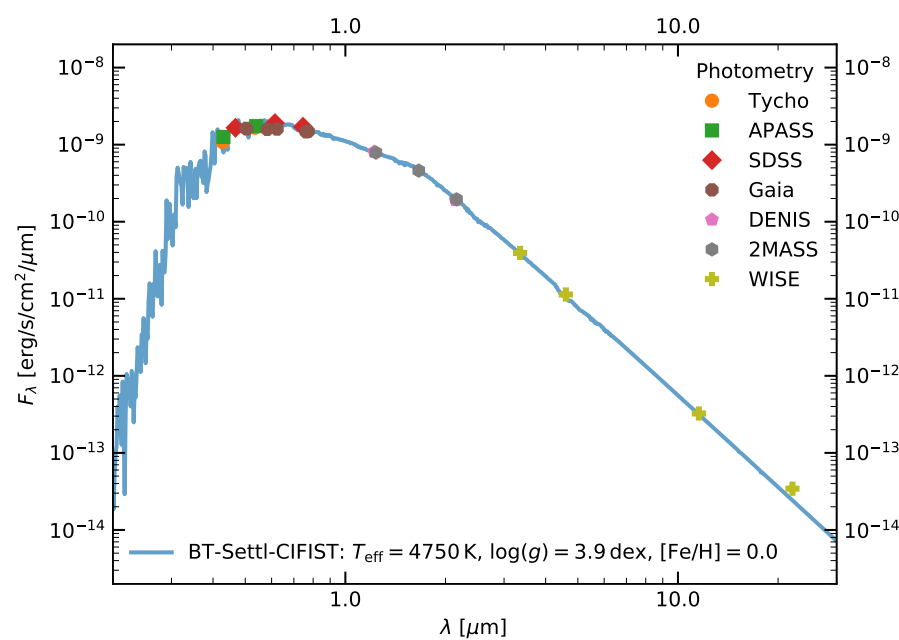

Fig. 1. Spectral energy distribution of YSES 2. The colored markers indicate the archival photometric measurements of the star and the blue curve presents our best-fit BT-Settl-CIFIST model to the data. The uncertainties of the photometric measurements are too small to be visualized in the figure.

$\left(\log \left(L / L_{\odot}\right)=-0.06\left(\right.\right.$ Preibisch \& Mamajek 2008), $\log \left(L / L_{\odot}\right)=$ $-0.265 \pm 0.075$ (Pecaut \& Mamajek 2016)) and benefits from a very precise distance, well-constrained extinction from $3 \mathrm{D}$ reddening maps, and integrating synthetic SEDs using 22 photometric data points. Combining this improved luminosity estimate with the improved $T_{\text {eff }}$ from the SED fitting ( $T_{\text {eff }}=$ $4749 \pm 40 \mathrm{~K}$ ) yields a good estimate of the radius of the star $\left(1.193 \pm 0.022 R_{\odot}\right)$. A comparison against evolutionary tracks from Baraffe et al. (2015) provided an updated stellar mass of $(1.10 \pm 0.03) M_{\odot}$ and an age of $(13.9 \pm 2.3)$ Myr. Furthermore we note that the SED of the star showed no signs of infrared excess through to the WISE-4 band $(22 \mu \mathrm{m})$. The 2MASSWISE colors $\left(K_{\mathrm{s}}-W 1=-0.035 \pm 0.032 \mathrm{mag}, K_{\mathrm{s}}-W 2=\right.$ $-0.007 \pm 0.030 \mathrm{mag}, K_{\mathrm{s}}-W 3=0.100 \pm 0.035 \mathrm{mag}, K_{\mathrm{s}}-W 4=$ $0.429 \pm 0.122 \mathrm{mag})$ can be compared to the mean for $\mathrm{K} 1$ premain-sequence stars from Pecaut \& Mamajek (2013) $\left(K_{\mathrm{s}}-W 1=\right.$ $0.09 \mathrm{mag}, K_{\mathrm{s}}-W 2=0.06 \mathrm{mag}, K_{\mathrm{s}}-W 3=0.10 \mathrm{mag}, K_{\mathrm{s}}-W 4=$ $0.18 \mathrm{mag}$ ), and show a significant hint of IR excess. The $K_{\mathrm{s}}-W 4$ color is marginally red ( $2 \sigma$ excess), perhaps hinting at a debris disk (common among non-accreting pre-main-sequence ScoCen stars), but there is no corroborating evidence to further support this.

We searched for common proper motion stellar or substellar companions for YSES 2. Assuming a mass of $1.0 M_{\odot}$, the tidal radius of YSES 2 is $\sim 1.35 \mathrm{pc}$ (projected radius $\sim 0^{\circ} .71$ or $\sim 2560^{\prime \prime}$; Mamajek et al. 2013), that is, bound companions would be expected to lie projected within this radius. Surveying the lists of Sco-Cen candidates and pre-main-sequence stars from the Gaia DR2 catalogs of Goldman et al. (2018), Zari et al. (2018), and Damiani et al. (2019) with parallactic distances of $<140 \mathrm{pc}$ shows only a couple of likely LCC siblings within $1^{\circ}$ of YSES 2: the poorly studied classical T Tauri star Wray 15-813 at 2845" ( $D=101 \mathrm{pc}$ ) (Pereira et al. 2003) and uncharacterized candidate pre-main-sequence object 2MASS J11375287-6631197 $(D=104 \mathrm{pc})$. A query of the recently released Gaia EDR3 catalog searching for co-moving, co-distant objects (with generous selection range of proper motions in $\alpha$ and $\delta$ within \pm 5 mas yr$^{-1}$, and parallax \pm 2 mas of YSES 2) yields zero candidate companions within $1^{\circ}$. Thus far, YSES 2 appears to be a stellar singleton. 

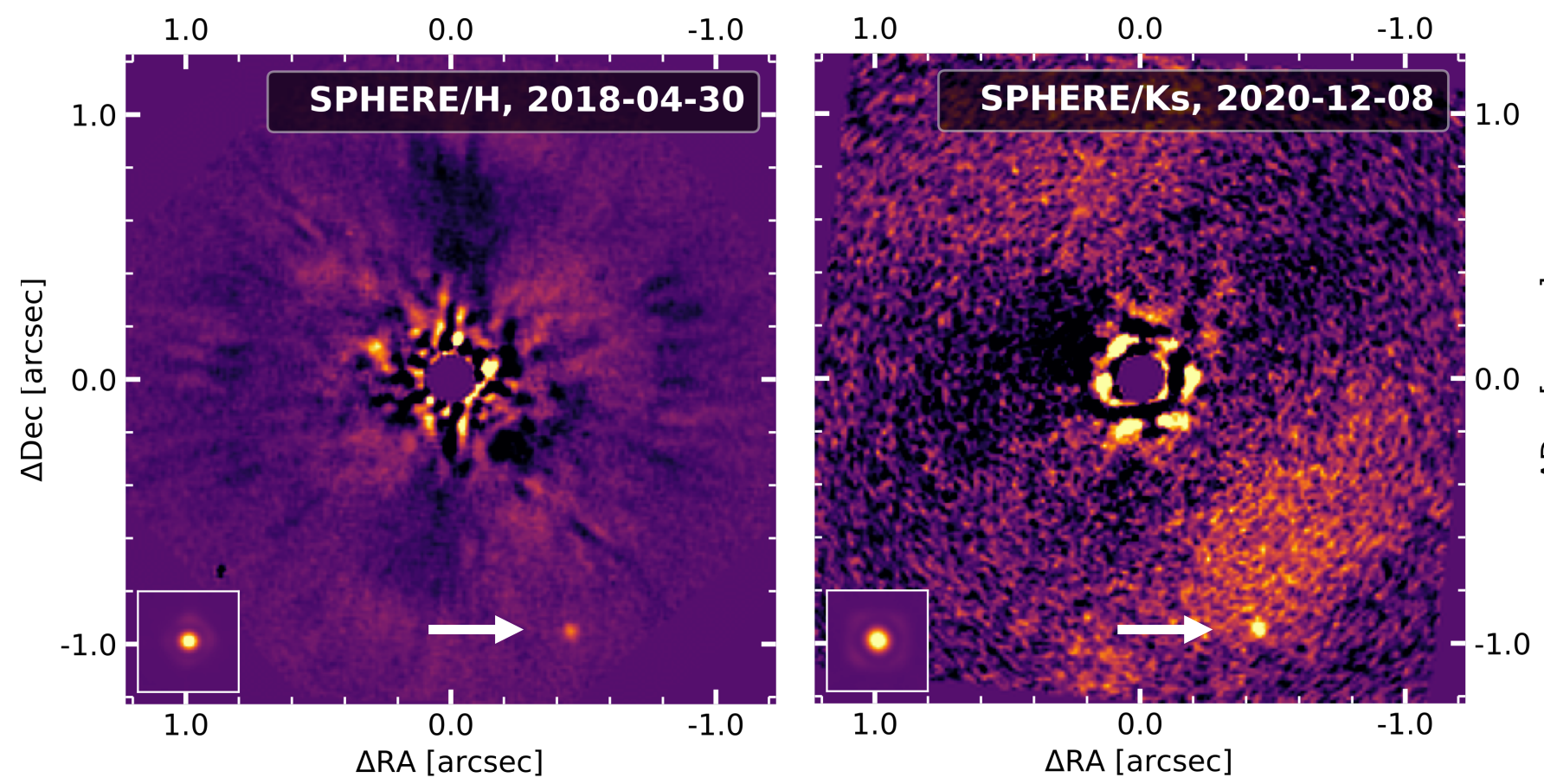

Fig. 2. Multi-epoch observations of YSES 2 and its planetary-mass companion. Final data products of the SPHERE observations collected in the $H$ band (left panel) and $K_{\mathrm{S}}$ band (right panel) are presented. For both filters, the stellar PSF is modeled by 50 principal components that were derived from a reference library of YSES targets. These PSF models were subtracted and the residuals rotated such that north points up and east toward the left. In the presented images the median of these de-rotated residuals is shown. For the $K_{\mathrm{s}}$ band data, uncorrected residuals of a wind-driven halo are detected that extend from the northeast to southwest. The planet YSES $2 b$ is highlighted by white arrows. The primary is located at the origin of the coordinate system and we artificially masked the inner region up to the radial extent of the coronagraphic mask of 100 mas. To assess the spatial extent of the instrumental PSF, the median combination of the non-coronagraphic flux images of the primary star are shown in the lower left of each panel. The intensity of each flux image is rescaled to match the maximum and minimum counts in the corresponding residual science image, and we display both images with the same spatial and color scales.

Table 2. Astrometry, photometry, and derived masses of YSES $2 b$.

\begin{tabular}{llllllll}
\hline \hline Observation date & Filter & Separation & Position angle & $\Delta$ Mag & \multicolumn{2}{c}{$M_{\text {abs }}$} & \multicolumn{2}{c}{$\begin{array}{c}\text { Photometric mass } \\
\text { COND } \\
\text { Dusty } \\
(\text { yyyy-mm-dd) }\end{array}$} & & $($ mas $)$ & $\left({ }^{\circ}\right)$ & $(\mathrm{mag})$ & $(\mathrm{mag})$ & $\left(M_{\text {Jup }}\right)$ & $\left(M_{\text {Jup }}\right)$ \\
\hline $2018-04-30$ & $H$ & $1057 \pm 3$ & $205.3 \pm 0.2$ & $10.37 \pm 0.11$ & $13.57 \pm 0.11$ & $5.3 \pm 0.5$ & $8.0 \pm 0.7$ \\
$2020-12-08$ & $K_{\mathrm{s}}$ & $1053 \pm 5$ & $205.2 \pm 0.2$ & $9.55 \pm 0.11$ & $12.71 \pm 0.11$ & $6.1 \pm 0.6$ & $6.4 \pm 0.6$ \\
\hline
\end{tabular}

\section{Observational results and analysis}

In Sect. 5.1, we show that our observations reveal a co-moving companion to YSES 2. The reduced images for both epochs are presented in Fig. 2. Our photometric analysis in Sect. 5.2 indicates that this companion has a mass that is significantly lower than the deuterium burning limit of $\sim 13 M_{\text {Jup }}$. We refer to this newly identified planet as YSES $2 b$ henceforth. In Sect. 5.3, we present the detection and mass limits of our acquired data.

\subsection{Companion astrometry}

We extracted the companion astrometry and photometry by the injection of negative artificial companions (e.g., Lagrange et al. 2010; Bonnefoy et al. 2011). A detailed description of our method is presented in Appendix C. The extracted astrometry is listed in Table 2. As visualized in the proper motion plot in Fig. 3, the companion is clearly incompatible with the calculated trajectory of a static background object at $14 \sigma$ significance. The relative astrometric motion with respect to the primary is consistent with a comoving companion. This conclusion is further confirmed by a similar analysis of other point sources within the detector field of view. As presented in Appendix D, all additional off-axis point sources are consistent with being non-moving background contaminants. We thus conclude that YSES $2 b$ is a gravitationally bound companion to its solar-mass host star. From our astrometric measurements we derived a projected physical separation of approximately 115 au. Future astrometric measurements are required to derive meaningful constraints to the orbital parameters of this wide-orbit planet.

\subsection{Companion photometry}

We present the photometry of the companion in Fig. 4 in a color-magnitude diagram. The corresponding numerical values are reported in Table 2 . YSES $2 \mathrm{~b}$ is consistent with a late $\mathrm{L}$ to early T spectral type when comparing it to colors of field brown dwarfs from the NIRSPEC Brown Dwarf Spectroscopic Survey 


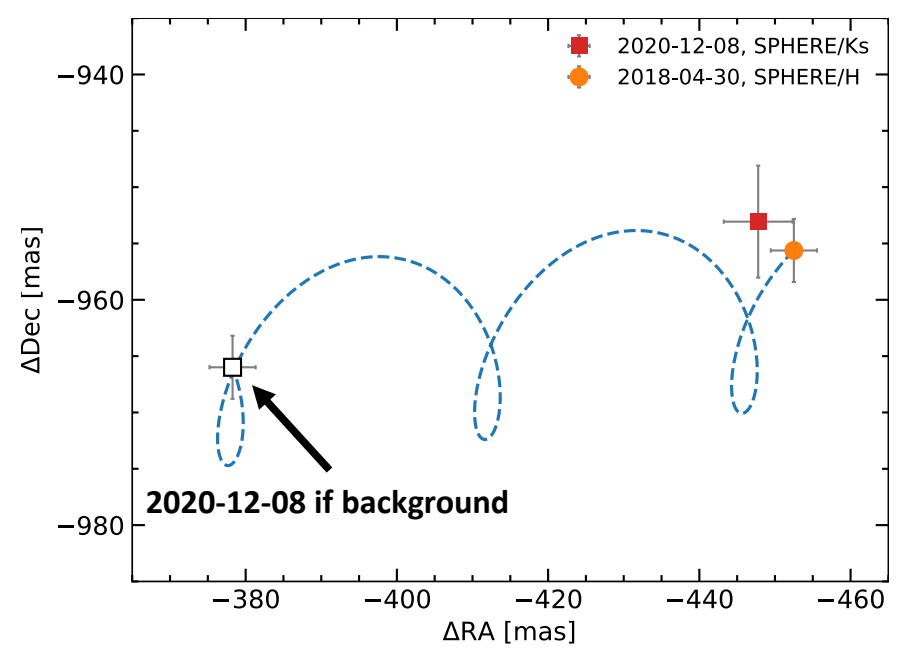

Fig. 3. Proper motion plot for YSES $2 b$. The colored markers represent the relative astrometry with respect to the primary star measured for our two observational epochs. The blue trajectory indicates the simulated motion of a static background object at infinity and the white marker is the theoretical position of such an object at our second observational epoch.

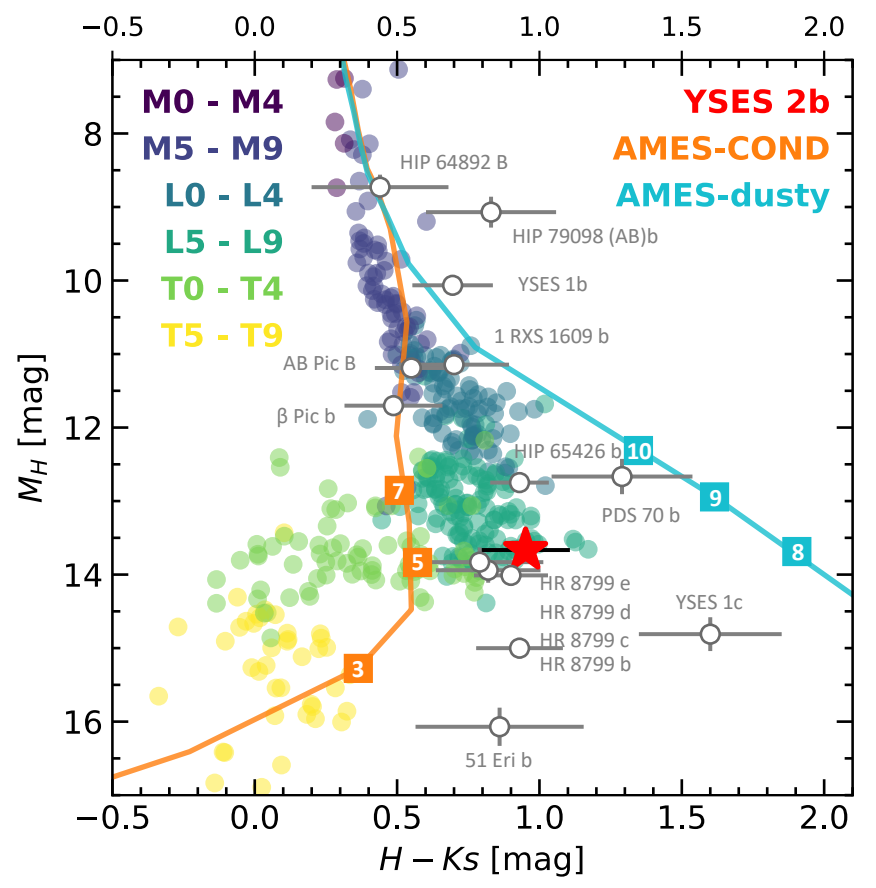

Fig. 4. Color-magnitude diagram for YSES 2b. The colored markers show the sequence of field brown dwarfs with various spectral types from $\mathrm{M}$ to late $\mathrm{T}$. The white markers represent known directly imaged companions that are usually younger than the presented field objects. YSES $2 b$ is highlighted by the red star. We further show AMES-COND and AMES-dusty evolutionary models that were evaluated at a system age of $13.9 \mathrm{Myr}$ (solid lines). The markers along the line indicate the equivalent object masses in $M_{\text {Jup }}$.

(McLean et al. 2003, 2007), the IRTF spectral library (Rayner et al. 2009; Cushing et al. 2005), the L and T dwarf data archive (Knapp et al. 2004; Golimowski et al. 2004; Chiu et al. 2006), and the SpeX Prism Libraries (Burgasser et al. 2004, 2008, 2010; Gelino \& Burgasser 2010; Burgasser 2007; Siegler et al. 2007; Reid et al. 2006; Kirkpatrick et al. 2006, 2010; Cruz et al. 2004; Burgasser \& McElwain 2006; McElwain \& Burgasser 2006;
Sheppard \& Cushing 2009; Looper et al. 2007, 2010; Muench et al. 2007; Dhital et al. 2011). Object distances were derived from Gaia EDR3 (Gaia Collaboration 2021), the Brown Dwarf Kinematics Project (Faherty et al. 2009), and the Pan-STARRS1 $3 \pi$ Survey (Best et al. 2018). In color-magnitude space, YSES $2 b$ is very close to the innermost three planets of the HR 8799 multiplanetary system (Marois et al. 2008, 2010). These three planets are classified as mid to late $L$ type dwarfs (e.g., Greenbaum et al. 2018), which agrees well with the sequence evolution of the adjacent field brown dwarfs from $\mathrm{L}$ to $\mathrm{T}$ spectral types ${ }^{3}$. A similar spectral type in this domain, therefore, seems very likely for YSES $2 b$, requiring confirmation by measurements at higher spectral resolution. Whereas the masses of the spectrally similar trio of HR $8799 \mathrm{c}, \mathrm{d}$, and e are in the range 7-12 $M_{\text {Jup }}$ (Wang et al. 2018; Marois et al. 2008, 2010), it is likely that YSES 2b has an even lower mass as the system age of $(13.9 \pm 2.3)$ Myr is significantly younger than the age of HR 8799 , which is claimed to be member of the Columba association with an age of 30-50 Myr (Zuckerman et al. 2011; Bell et al. 2015). This is supported by the AMES-COND and AMES-dusty models (Allard et al. 2001; Chabrier et al. 2000) that we present in Fig. 4 for a system age of 13.9 Myr. An individual evaluation of these isochrones yielded masses from $5.3 M_{\mathrm{Jup}}$ to $8.0 M_{\mathrm{Jup}}$ as presented in Table 2 . The uncertainties originate from the errors in the system age and planet magnitude that were propagated by a bootstrapping approach with 1000 randomly drawn samples from Gaussian distributions around both parameters. When combining the posterior distributions for the different models and filters we derived a final mass estimate of $6.3_{-0.9}^{+1.6} M_{\text {Jup }}$ as the $68 \%$ confidence interval around the median of the sample. This estimate is based on broadband photometric measurements alone; further spectral coverage of the planetary SED will be important to constrain its effective temperature, luminosity, surface gravity, and mass.

\subsection{Detection limits}

To derive upper mass limits for additional companions in the system, we calculated the detection limits of our datasets. As a baseline, we evaluated the contrast in the image that was obtained by de-rotating and median combining the individual exposures without any PSF subtraction. This image covers the full field of view of the SPHERE/IRDIS detector up to an angular separation of 5'.5. We evaluated the contrast directly in the final image using aperture photometry. The chosen aperture size was one full width at half maximum (FWHM) of the unsaturated stellar PSF as measured in the flux images (see Table A.1). The signal flux was measured as the sum over the full circular aperture within the mean combined flux image and scaled for the flux difference with the science frames owing to the shorter exposure times and the applied neutral density filters. For several radial positions that were equidistantly sampled from $0 . ' 15$ to $5^{\prime \prime} .50$ in steps of $0 . ' 05$, we measured the noise as the standard deviation of the integrated flux within apertures that were distributed around the star at the same radial separation (excluding the signal aperture itself). We applied a sigma clipping with an upper bound of $3 \sigma$ to the integrated fluxes of the noise apertures before calculating the standard deviation to discard apertures that were polluted by flux of off-axis point sources (see the full frame image in Fig. D.1). A correction for small sample statistics as

\footnotetext{
3 We would like to note that the planets around HR 8799, although closely located to the sequence of field brown dwarfs in the selected SPHERE filters as presented in the color-magnitude diagram in Fig. 4, can have near-infrared colors in different passbands that are significantly distinct from those of their field dwarf analogs (e.g., Currie et al. 2011).
} 

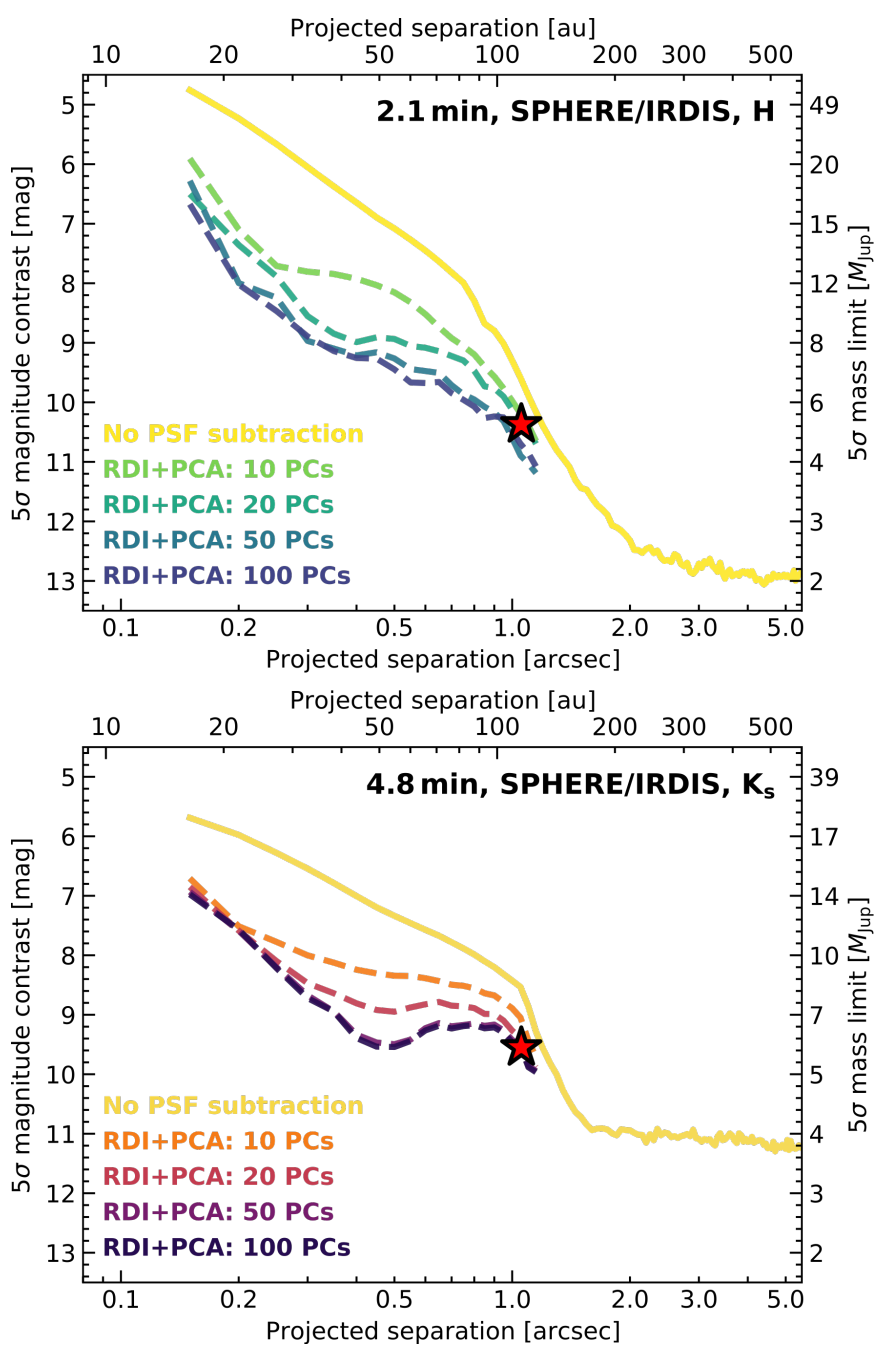

Fig. 5. $5 \sigma$ detection limits of our SPHERE/IRDIS observations in the $H$ (upper panel) and $K_{\mathrm{s}}$ bands (lower panel). On the left axis the magnitude contrast with respect to the primary star is reported, and the absolute magnitudes are converted to detectable planet masses with AMES-COND models as indicated on the right axis; this scale varies between the $H$ and $K_{\mathrm{s}}$ bands. The solid yellow lines represent the limits when no PSF subtraction is performed. The dashed lines indicate the sensitivity, when a PSF subtraction with RDI plus PCA is performed. The red star highlights the contrast of YSES $2 \mathrm{~b}$ that we detect at $\sim 5 \sigma$ significance in both filters after PSF subtraction with more than 50 principal components.

described in Mawet et al. (2014) was considered in these noise calculations. We reiterated this analysis for six uniformly spaced position angles and present the azimuthally averaged results as two-dimensional contrast curves in Fig. 5. The solid yellow lines represent the $5 \sigma$ raw contrast in the $H$ and $K_{\mathrm{s}}$ bands that was obtained without any PSF subtraction.

For the innermost region around the star $\left(<1^{\prime \prime} .2\right)$, the sensitivity was additionally assessed considering our PSF subtraction by RDI plus PCA. We used the ContrastCurveModule from PynPoint version $0.6 .0^{4}$ that utilizes the same aperture

\footnotetext{
4 As mentioned before, version 0.8.1 of PynPoint was used for all remaining analysis steps. The modules of both versions are compatible; only the implementation of some algorithms changed throughout the development process. This affects the ContrastCurveModule, which follows the iterative process described in this paragraph for release version 0.6.0. We prefer this implementation over the solution presented
}

photometry framework and metric to evaluate the contrast for several positions that were distributed around the star in the residual images. For each position, the module injects an artificial companion, whose detection significance is evaluated after the PSF subtraction with RDI combined with PCA. In this framework, the signal aperture is directly placed on top of the position at which the artificial companion has been injected, and the noise apertures are azimuthally distributed around the primary star as described before, yet excluding the signal aperture itself. The companion template was obtained as the median combination of the non-coronagraphic flux images that was scaled for the difference in exposure time and the attenuation due to the applied neutral density filter. From an initial magnitude contrast of $8 \mathrm{mag}$, the flux of the injected companion was adjusted and the post-processing was performed iteratively until the artificial companion was retrieved at $5 \sigma$ detection significance in the final image product. These limiting magnitude contrasts were stored for each of the injection positions. For the calculation of the final contrast curves with RDI plus PCA, we used a radial sampling in the range $0 . ' 15-1{ }^{\prime \prime} .20$ with a spatial resolution of 0.05 , and six position angles that were equidistantly sampled in polar space. Again, the contrast as a function of radial separation was obtained by azimuthal averaging of the various position angles. We considered several numbers of principal components to model the stellar PSF as indicated by the sequentially colored, dashed lines in Fig. 5. The detectable planet masses that correspond to the calculated magnitude contrasts were derived by evaluation of AMES-COND models at the system age of 13.9 Myr (see right axes of Fig. 5).

The contrast performance close the star improves for an increasing number of principal components. This differential gain in contrast ceases for $\sim 50$ subtracted components and the contrast for 100 principal components does not change significantly compared to the curve generated for half as many components. This justifies our previously selected value of 50 principal components that were used for our PSF subtraction with RDI plus PCA. This amount of components is equivalent to $19 \%$ and $30 \%$ of the reference libraries in the $H$ and $K_{\mathrm{s}}$ bands, which are composed of 269 and 164 individual frames, respectively. In the $H$ band we observe a contrast improvement of more than two magnitudes at an angular separation of 0.2 . This corresponds to an increase in planet detection sensitivity by more than $45 M_{\text {Jup }}$ at this close separation. The contrast improvement in the $H$ band is maximized at an angular separation of $\sim 0 .^{\prime} 3$, where RDI plus PCA provides detection limits that are approximately three magnitudes deeper than our raw data. At angular separations larger than $1^{\prime \prime}$ the contrast improvement decreases as the flux contribution of the stellar PSF becomes negligible. At separations $\geq 2$ " we reach a fundamental noise floor that is mainly composed of residual sky background and detector read out noise. The $K_{\mathrm{s}}$ band contrast behaves very similar to the detection limits in the $H$ band and the RDI plus PCA reduction scheme can provide a maximum gain of up to $2.5 \mathrm{mag}$ at an angular separation of $\sim 0$.' 4 . The overall improvement for separations $<1^{\prime \prime}$ is marginally worse compared to the $H$ band data and the contribution of the asymmetric wind-driven halo is clearly visible for separations in the range $0.5-1{ }^{\prime \prime} 2$. Combining the data from the $H$ and $K_{\mathrm{s}}$ bands allows us to exclude stellar and brown dwarf companions around YSES 2 with masses $>13 M_{\text {Jup }}$ for angular separations that are larger than $0{ }^{\prime} 15$. At $0{ }^{\prime} 5$ we are sensitive to

in PynPoint version 0.8.1, which calculates one attenuation factor per position that is based on the single injection of an artificial companion with a user-defined signal-to-noise ratio. 
objects that are more massive than $6 M_{\text {Jup }}$ and for angular separations that are larger than $2^{\prime \prime}$ we can even rule out planets with masses as low as $2 M_{\text {Jup }}$.

This demonstrates, impressively, how a large reference library can help to significantly improve the contrast performance at small angular separations $<1$ '.2 2 . Especially for datasets with little parallactic rotation, RDI plus PCA should be considered as a default PSF subtraction strategy. This conclusion is also supported by first results from the star-hopping mode that was recently implemented at VLT/SPHERE (Wahhaj et al. 2021). As visualized by the red stars in Fig. 5, RDI plus PCA is required to detect YSES $2 \mathrm{~b}$ at $5 \sigma$ significance in both the $H$ and $K_{\mathrm{s}}$ band data.

\section{Discussion}

The newly discovered planetary companion to YSES 2 is among the lowest mass direct imaging companions known to date. The only objects of similar low mass are 51 Erib (Macintosh et al. 2015), HD 95086 b (Rameau et al. 2013), HR8799 b (Marois et al. 2008), PDS $70 \mathrm{~b}$ and c (Keppler et al. 2018; Haffert et al. 2019; Wang et al. 2020; Stolker et al. 2020a), and YSES 1c (Bohn et al. 2020b). Of these, only YSES $1 \mathrm{c}$ is located around a solarmass star. Within the uncertainties, the mass of YSES $2 b$ is the same as YSES 1c, which we previously discovered in our survey. Given our mass estimate for the planet, the mass ratio of YSES $2 \mathrm{~b}$ to its host star is $q=0.54_{-0.08}^{+0.13} \%$. This value is comparable, but slightly lower than the $q=0.57 \pm 0.10 \%$ derived for YSES 1c. The mass ratio is the lowest among direct imaging companions to solar-type stars 5 .

The in situ formation of super-Jovian planets at tens or hundreds of astronomical units is challenging. We recently discussed possible formation scenarios for such objects in the context of the YSES 1 system in Bohn et al. (2020a). In our previous study we considered scattering or planet capturing events to explain the current large separation of YSES 1b. However, dynamic scattering by a third body in the system is expected to produce high eccentricities, inconsistent with orbital stability of both planetary components in the YSES 1 system (Bohn et al. 2020b) ${ }^{6}$. The new detection of YSES $2 b$ in our small survey sample of 70 solar-type systems in Sco-Cen makes the hypothesis that we see captured free-floating planets unlikely. Goulinski \& Ribak (2018) find with numerical simulations that only $\sim 0.1 \%$ of solartype stars in the Galactic thin disk should capture a free-floating planet in their lifetime. YSES $2 b$ in principle might have formed in situ via disk gravitational instability. Boss (2011) find that they can produce $1-5 M_{\text {Jup }}$ planets between $30 \mathrm{au}$ and $70 \mathrm{au}$ with eccentricities as high as 0.35 . If YSES $2 b$ is on an eccentric orbit it may explain its current projected separation of $110 \mathrm{au}$. Kratter et al. (2010) conversely find with their hydrodynamic simulations that planets formed via disk instability need to be at large separations outside of the $40 \mathrm{au}$ to $70 \mathrm{au}$ range to not accumulate too much mass and remain in the planetary regime. This hypothesis that gravitationally instabilities predominantly create brown dwarf and stellar companions is supported by other theoretical studies (e.g., Zhu et al. 2012; Forgan \& Rice 2013). Spatially resolved observations of gas-rich planet forming disks in the last few years have shown that radial substructures, which

\footnotetext{
5 See Bohn et al. (2020a) for an overview of mass ratios of direct imaging companions to solar-type stars.

6 We, however, note that in some cases high eccentricities are inferred for substellar companions, for example, the brown dwarf companion to the young solar analog PZ Tel (Mugrauer et al. 2012; Ginski et al. 2014).
}

are thought to be caused by perturbing planets, are nearly ubiquitous (see, e.g., Garufi et al. 2018 for an overview, observed in scattered light). These structures can, in a growing number of cases, be traced out to tens or hundreds of astronomical units (e.g., ALMA Partnership 2015; Ginski et al. 2016; de Boer et al. 2016; van Terwisga et al. 2018). Recently it was found that these substructures are already present in proto-stellar disks as young as 0.1 Myr (Sheehan et al. 2020; Sheehan 2020), suggesting that planet formation sets in early and operates on short timescales. Despite this abundance of substructures observed in young, circumstellar environments, it is unlikely that all these protoplanetary disks are gravitationally unstable and support planet formation via this channel (e.g., Kratter \& Lodato 2016). Conversely, we expect that the timescale to form a planet via core accretion at the current location of YSES $2 b$ would be too long, given the system age and that the gas-rich disk in the system has already dissipated (e.g., Haisch et al. 2001).

Even though capturing scenarios are considered unlikely for YSES $2 b$, we cannot confidently conclude whether its formation via either top-down or bottom-up scenarios is more likely: whereas a mass of $6.3 M_{\text {Jup }}$ is rather low for an object to originate from gravitational disk instabilities, core accretion would favor a formation at closer separations to the star. More data are thus required to explore the origin of this wide-orbit Jovian giant. Promising methods to evaluate the likelihood of either formation scenario are by characterization measurements of the planetary atmosphere, continuous orbital monitoring to constrain especially its eccentricity, and deeper searches for additional companions in the system.

To identify the formation channel of YSES $2 b$ via atmospheric characterization, we can utilize the framework postulated by Öberg et al. (2011), who argued that elemental abundances in the planetary atmosphere (and especially the $\mathrm{C} / \mathrm{O}$ ratio) are directly linked to its natal environment in the planet-forming disk. Different ice lines in the protoplanetary disk and the associated freeze out of the corresponding molecular species alter molecular abundance ratios as a function of radial separation from the host star, making this atmospheric quantity a promising indicator of the natal environment and formation channel of a planet. The chemical and dynamical evolution of the disk can alter these initial abundances and should be considered in the analysis (e.g., Ali-Dib et al. 2014; Mordasini et al. 2016; Eistrup et al. 2016, 2018). Gravity Collaboration (2020) utilized this framework to study $\beta$ Pic $\mathrm{b}$ and proposed its formation through core accretion, with strong planetesimal enrichment based on its subsolar $\mathrm{C} / \mathrm{O}$ abundance ratio. If YSES $2 b$ formed via disk gravitational instability, then we expect this object to have similar elemental abundances as the primary star in the system, while formation by core accretion should lead to an overabundance of heavy elements due to pebble accretion. YSES $2 b$, along with other planet mass objects detected by direct imaging, provides an ideal test case for future detailed atmospheric characterization.

We can further continue to monitor the separation and position angle of YSES $2 b$ with respect to the primary star to derive orbital solutions for the planet (e.g., Wang et al. 2018). In particular, the VLTI/GRAVITY instrument (Gravity Collaboration 2017) will be extremely useful for this purpose, as it facilitates an unprecedented astrometric precision down to sub-milliarcsecond scales (e.g., Gravity Collaboration 2019; Wang et al. 2021). These astrometry measurements could be complemented by VLT/CRIRES $^{+}$data to constrain the radial velocity of the planet and to obtain three-dimensional information about its orbital motion (e.g., Schwarz et al. 2016). The eccentricity of the planet 
might provide hints regarding the likelihood of a potential migration of the companion, which would be an indicator of formation via core accretion at closer separation to the star. If this migration was caused by scattering off another, so far undetected companion to the primary star, a deep imaging campaign is required to search for evidence of such an additional component to the planetary system. At the moment, we cannot provide conclusive evidence for the most likely formation scenario of YSES $2 b$ based on the available data; but future observations might be able to shed light on the origin of this Jovian gas giant.

Even though the second epoch observations of YSES are not concluded yet and candidate companions to $\sim 45$ stars of our sample need to be confirmed or rejected, our survey has already discovered three planetary-mass companions amongst 70 young, Sun-like stars. This high planet-detection rate is in stark contrast to previous surveys that were targeting Sun-like stars at closer distances than the LCC (e.g., Kasper et al. 2007; Biller et al. 2013; Galicher et al. 2016), which discovered mostly stellar and brown dwarf companions. These preliminary statistical results from YSES tentatively indicate that despite the farther distance, Sco-Cen and especially LCC are more favorable than moving groups in the immediate solar neighborhood for the detection of young planets briefly after their formation. Many of the moving groups that were targeted in these aforementioned surveys are significantly older than LCC $(15 \pm 3 \mathrm{Myr})$, such as the TucanaHorologium moving group ( $45 \pm 4 \mathrm{Myr}$; Bell et al. 2015), the AB Dor moving group (149 ${ }_{-19}^{+51} \mathrm{Myr}$; Bell et al. 2015), or the Hercules-Lyra association (257 \pm 46 Myr; Eisenbeiss et al. 2013). As a consequence of the decreasing luminosity of objects below the deuterium burning limit with increasing age (e.g., Burrows et al. 1997), it is natural that the sensitivity to Jovian planets is worse around members of these associations compared to significantly younger host stars. Yet some of these closer moving groups have ages comparable to that of LCC - such as the TW Hya association (10 $\pm 3 \mathrm{Myr})$ or the $\beta$ Pic moving group ( $24 \pm 3$ Myr; Bell et al. 2015) - and should provide even better planet-detection sensitivities owing to their much closer distances. However, before speculating about potential reasons for this tentative overabundance of planetary-mass companions to our YSES targets, it is necessary to finish the second epoch observations of the survey and to derive reliable occurrence rates of planetary-mass companions to Sun-like stars in Sco-Cen.

\section{Conclusions}

We report the detection of a new directly imaged planet to the solar-mass primary YSES 2 that was discovered within the scope of YSES. Reassessment of the stellar parameters provided an effective temperature of $T_{\text {eff }}=(4749 \pm 40) \mathrm{K}$, a luminosity of $\log \left(L / L_{\odot}\right)=-0.1854 \pm 0.0063$, a mass of $(1.10 \pm 0.03) M_{\odot}$, and a system age of $(13.9 \pm 2.3)$ Myr.

We detect YSES $2 b$ in two consecutive epochs collected on 2018 April 30 and 2020 December 18 with VLT/SPHERE. The companion has a projected separation of approximately 1 .'05, which translates to a physical minimum distance of $\sim 115$ au with respect to the primary star. Photometric measurements in the $H$ and $K_{\mathrm{s}}$ bands constrain a planet mass of $6.3_{-0.9}^{+1.6} M_{\text {Jup }}$ according to AMES-COND and AMES-dusty evolutionary models. This mass estimate is supported by the position of the object in colormagnitude space, where it is located amongst the mid to late $L$ type field brown dwarfs and close to HR 8799 c, d, and e. The slightly higher mass estimates of these exoplanets on the order of 7-12 $M_{\text {Jup }}$ are consistent with the older system age of HR 8799 of 30-50 Myr.
The mass and separation of YSES $2 b$ are inconsistent with planet populations for most in situ formation scenarios: whereas disk instabilities predominantly create companions above the deuterium burning limit at a separation of $110 \mathrm{au}$, core-accretion mechanisms are not efficient enough to form a planet of $6.3 M_{\text {Jup }}$ this widely separated from the primary star. So, the new companion might be either at the low-mass end of potential in situ formation outcomes from top-down scenarios, or it formed via core accretion at closer separation to the star and migrated to its current location. Atmospheric characterization measurements of molecular abundance ratios, orbital monitoring, and evaluation of the eccentricity of the planet, or a deep search for additional companions in the system, might help to evaluate the likelihood of these potential formation pathways. While we cannot rule out scattering or capture scenarios, we point out that the former require an (as of yet) third undetected body in the system, while the latter are unlikely given numerical simulations. YSES $2 b$ is an important addition to the sparsely populated group of wide-orbit gas giant companions. Owing to the moderate separation with respect to the primary star, spectroscopic observations with JWST, VLT/ERIS, or VLTI/GRAVITY will be easily available. These data will be important to further constrain the properties of this Jovian companion. Measurements of molecular abundance ratios such as $\mathrm{C} / \mathrm{O}$ or its orbital eccentricity might even facilitate hypotheses regarding the most likely formation mechanism for this wide-orbit gas giant planet.

Our data rule out brown dwarf and stellar companions with $M>13 M_{\text {Jup }}$ in the SPHERE/IRDIS field of view for angular separations $>0$.' 15 and at 0.5 we can exclude objects that are more massive than $6 M_{\text {Jup }}$. At separations that are larger than $2^{\prime \prime}$ we are even sensitive to planets with masses as low as $2 M_{\text {Jup }}$. In general, the applied PSF subtraction scheme based on RDI plus PCA is extremely successful and provides substantial contrast improvements $(>1 \mathrm{mag})$ for separations that are smaller than $1^{\prime \prime}$. In the $H$ band, the PSF subtraction enhances our sensitivity by more than $45 M_{\text {Jup }}$ at 0.2 , and the greatest contrast improvement of $\sim 3 \mathrm{mag}$ is achieved at an angular separation of 0.'3. Our YSES strategy with short snapshot observations of $\leq 5$ min combined with a large reference library for PSF subtraction is certainly a promising approach to image planetary-mass companions to young, Sun-like stars in Sco-Cen. With three newly discovered planetary-mass companions in less than $40 \mathrm{~h}$ of allocated telescope time the survey efficiency is unprecedented and the mission concept can certainly be applied to future high-contrast imaging studies targeting different samples of pre-main-sequence stars.

Acknowledgements. We would like to thank the anonymous referee for the very valuable feedback that helped improving the quality of the manuscript. Especially, the extremely kind way of providing this feedback was highly appreciated by the authors. The research of A.J.B. and F.S. leading to these results has received funding from the European Research Council under ERC Starting Grant agreement 678194 (FALCONER). Part of this research was carried out at the Jet Propulsion Laboratory, California Institute of Technology, under a contract with the National Aeronautics and Space Administration (80NM0018D0004). M.M. would like to thank the German Research Foundation (DFG) for support in the program MU 2695/27-1. M.R. acknowledges support from the FWO research program under project $1280121 \mathrm{~N}$. This research has used the SIMBAD database, operated at CDS, Strasbourg, France (Wenger et al. 2000). This work has used data from the European Space Agency (ESA) mission Gaia (https://www. cosmos.esa.int/gaia), processed by the Gaia Data Processing and Analysis Consortium (DPAC, https://www.cosmos. esa.int/web/gaia/dpac/consortium). Funding for the DPAC has been provided by national institutions, in particular the institutions participating in the Gaia Multilateral Agreement. This publication makes use of VOSA, developed under the Spanish Virtual Observatory project supported by the Spanish MINECO through grant AyA2017-84089. To achieve the scientific results presented in this article we made use of the Python programming language (Python 
Software Foundation, https://www.python.org/), especially the SciPy (Virtanen et al. 2020), NumPy (Oliphant 2006), Matplotlib (Hunter 2007), emcee (Foreman-Mackey et al. 2013), scikit-image (Van der Walt et al. 2014), scikitlearn (Pedregosa et al. 2012), photutils (Bradley et al. 2016), and astropy (Astropy Collaboration 2013, 2018) packages.

\section{References}

Alibert, Y., Mordasini, C., Benz, W., \& Winisdoerffer, C. 2005, A\&A, 434, 343

Ali-Dib, M., Mousis, O., Petit, J.-M., \& Lunine, J. I. 2014, ApJ, 785, 125

Allard, F., Hauschildt, P. H., Alexander, D. R., Tamanai, A., \& Schweitzer, A. 2001, ApJ, 556, 357

ALMA Partnership (Brogan, C. L., et al.) 2015, ApJ, 808, L3

Amara, A., \& Quanz, S. P. 2012, MNRAS, 427, 948

Astropy Collaboration (Robitaille, T. P., et al.) 2013 A\&A, 558, A33

Astropy Collaboration (Price-Whelan, A. M., et al.) 2018, AJ, 156, 123

Bailey, V., Meshkat, T., Reiter, M., et al. 2014, ApJ, 780, L4

Baraffe, I., Homeier, D., Allard, F., \& Chabrier, G. 2015, A\&A, 577, A42

Bayo, A., Rodrigo, C., Barrado Y Navascués, D., et al. 2008, A\&A, 492, 277

Bell, C. P. M., Mamajek, E. E., \& Naylor, T. 2015, MNRAS, 454, 593

Best, W. M. J., Magnier, E. A., Liu, M. C., et al. 2018, ApJS, 234, 1

Beuzit, J. L., Vigan, A., Mouillet, D., et al. 2019, A\&A, 631, A155

Biller, B. A., Liu, M. C., Wahhaj, Z., et al. 2013, ApJ, 777, 160

Bohn, A. J., Kenworthy, M. A., Ginski, C., et al. 2019, A\&A, 624, A87

Bohn, A. J., Kenworthy, M. A., Ginski, C., et al. 2020a, MNRAS, 492, 431

Bohn, A. J., Kenworthy, M. A., Ginski, C., et al. 2020b, ApJ, 898, L16

Bonnefoy, M., Lagrange, A. M., Boccaletti, A., et al. 2011, A\&A, 528, A15

Boss, A. P. 1997, Science, 276, 1836

Boss, A. P. 2011, ApJ, 731, 74

Bowler, B. P. 2016, PASP, 128, 102001

Bowler, B. P., Blunt, S. C., \& Nielsen, E. L. 2020, AJ, 159, 63

Bradley, L., Sipocz, B., Robitaille, T., et al. 2016, Photutils: Photometry Tools

Burgasser, A. J. 2007, ApJ, 659, 655

Burgasser, A. J., \& McElwain, M. W. 2006, AJ, 131, 1007

Burgasser, A. J., McElwain, M. W., Kirkpatrick, J. D., et al. 2004, AJ, 127, 2856

Burgasser, A. J., Liu, M. C., Ireland, M. J., Cruz, K. L., \& Dupuy, T. J. 2008, ApJ, 681, 579

Burgasser, A. J., Cruz, K. L., Cushing, M., et al. 2010, ApJ, 710, 1142

Burrows, A., Marley, M., Hubbard, W. B., et al. 1997, ApJ, 491, 856

Cantalloube, F., Por, E. H., Dohlen, K., et al. 2018, A\&A, 620, A10

Carbillet, M., Bendjoya, P., Abe, L., et al. 2011, Exp. Astron., 30, 39

Chabrier, G. 2003, PASP, 115, 763

Chabrier, G., Baraffe, I., Allard, F., \& Hauschildt, P. 2000, ApJ, 542, 464

Chauvin, G., Desidera, S., Lagrange, A. M., et al. 2017, in SF2A-2017: Proceedings of the Annual meeting of the French Society of Astronomy and Astrophysics, (eds. C. Reylé, P. Di Matteo, F. Herpin, E., et al.) 331

Chiu, K., Fan, X., Leggett, S. K., et al. 2006, AJ, 131, 2722

Choquet, E., Hagan, J. B., Pueyo, L., et al. 2014, in Exploring the Formation and Evolution of Planetary Systems, 299, 30

Cruz, K. L., Burgasser, A. J., Reid, I. N., \& Liebert, J. 2004, ApJ, 604, L61

Currie, T., Burrows, A., Itoh, Y., et al. 2011, ApJ, 729, 128

Cushing, M. C., Rayner, J. T., \& Vacca, W. D. 2005, ApJ, 623, 1115

Cutri, R. M., Skrutskie, M. F., van Dyk, S., et al. 2003, 2MASS All Sky Catalog of Point Sources (IPAC)

Cutri, R. M., et al. 2014, VizieR Online Data Catalog, II/328

Damiani, F., Prisinzano, L., Pillitteri, I., Micela, G., \& Sciortino, S. 2019, A\&A, 623, A112

de Boer, J., Salter, G., Benisty, M., et al. 2016, A\&A, 595, A114

de Zeeuw, P. T., Hoogerwerf, R., de Bruijne, J. H. J., Brown, A. G. A., \& Blaauw, A. 1999, AJ, 117, 354

Dhital, S., Burgasser, A. J., Looper, D. L., \& Stassun, K. G. 2011, AJ, 141, 7

Dodson-Robinson, S. E., Veras, D., Ford, E. B., \& Beichman, C. A. 2009, ApJ, 707, 79

Dohlen, K., Langlois, M., Saisse, M., et al. 2008, in Proc. SPIE, 7014, Groundbased and Airborne Instrumentation for Astronomy II, 70143L

Durisen, R. H., Boss, A. P., Mayer, L., et al. 2007, in Protostars and Planets V, eds. B. Reipurth, D. Jewitt, \& K. Keil, 607

Eisenbeiss, T., Ammler-von Eiff, M., Roell, T., et al. 2013, A\&A, 556, A53

Eisenhardt, P. R. M., Marocco, F., Fowler, J. W., et al. 2020, ApJS, 247, 69

Eistrup, C., Walsh, C., \& van Dishoeck, E. F. 2016, A\&A, 595, A83

Eistrup, C., Walsh, C., \& van Dishoeck, E. F. 2018, A\&A, 613, A14

Faherty, J. K., Burgasser, A. J., Cruz, K. L., et al. 2009, AJ, 137, 1

Foreman-Mackey, D., Hogg, D. W., Lang, D., \& Goodman, J. 2013, PASP, 125, 306

Forgan, D., \& Rice, K. 2013, MNRAS, 432, 3168
Forgan, D. H., Hall, C., Meru, F., \& Rice, W. K. M. 2018, MNRAS, 474, 5036 Gagné, J., Mamajek, E. E., Malo, L., et al. 2018, ApJ, 856, 23

Gaia Collaboration (Brown, A. G. A., et al.) 2018, A\&A, 616, A1

Gaia Collaboration (Brown, A. G. A., et al.) 2021, A\&A, in press, https://doi.org/10.1051/0004-6361/202039657

Galicher, R., Marois, C., Macintosh, B., et al. 2016, A\&A, 594, A63

Garufi, A., Benisty, M., Pinilla, P., et al. 2018, A\&A, 620, A94

Gelino, C. R., \& Burgasser, A. J. 2010, AJ, 140, 110

Ginski, C., Schmidt, T. O. B., Mugrauer, M., et al. 2014, MNRAS, 444, 2280

Ginski, C., Mugrauer, M., Seeliger, M., et al. 2016, MNRAS, 457, 2173

Goldman, B., Röser, S., Schilbach, E., Moór, A. C., \& Henning, T. 2018, ApJ, 868,32

Golimowski, D. A., Leggett, S. K., Marley, M. S., et al. 2004, AJ, 127, 3516

Goulinski, N., \& Ribak, E. N. 2018, MNRAS, 473, 1589

Gravity Collaboration (Abuter, R., et al.) 2017, A\&A, 602, A94

Gravity Collaboration (Lacour, S., et al.) 2019, A\&A, 623, A11

Gravity Collaboration (Nowak, M., et al.) 2020, A\&A, 633, A110

Greenbaum, A. Z., Pueyo, L., Ruffio, J.-B., et al. 2018, AJ, 155, 226

Haffert, S. Y., Bohn, A. J., de Boer, J., et al. 2019, Nat. Astron., 3, 749

Haisch, Karl E., J., Lada, E. A., \& Lada, C. J. 2001, ApJ, 553, L153

Henden, A. A., Templeton, M., Terrell, D., et al. 2016, VizieR Online Data Catalog, II $/ 336$

Høg, E., Fabricius, C., Makarov, V. V., et al. 2000, A\&A, 355, L27

Hunter, J. D. 2007, Comput. Sci. Eng., 9, 90

Janson, M., Asensio-Torres, R., André, D., et al. 2019, A\&A, 626, A99

Johansen, A., \& Lacerda, P. 2010, MNRAS, 404, 475

Kasper, M., Apai, D., Janson, M., \& Brandner, W. 2007, A\&A, 472, 321

Keppler, M., Benisty, M., Müller, A., et al. 2018, A\&A, 617, A44

Kiraga, M. 2012, Acta Astron., 62, 67

Kirkpatrick, J. D., Barman, T. S., Burgasser, A. J., et al. 2006, ApJ, 639, 1120

Kirkpatrick, J. D., Looper, D. L., Burgasser, A. J., et al. 2010, ApJS, 190, 100

Knapp, G. R., Leggett, S. K., Fan, X., et al. 2004, AJ, 127, 3553

Kratter, K., \& Lodato, G. 2016, ARA\&A, 54, 271

Kratter, K. M., Murray-Clay, R. A., \& Youdin, A. N. 2010, ApJ, 710, 1375

Kroupa, P. 2001, MNRAS, 322, 231

Lagrange, A. M., Bonnefoy, M., Chauvin, G., et al. 2010, Science, 329, 57

Lallement, R., Babusiaux, C., Vergely, J. L., et al. 2019, A\&A, 625, A135

Lambrechts, M., \& Johansen, A. 2012, A\&A, 544, A32

Looper, D. L., Burgasser, A. J., Kirkpatrick, J. D., \& Swift, B. J. 2007, ApJ, 669, L97

Looper, D. L., Bochanski, J. J., Burgasser, A. J., et al. 2010, AJ, 140, 1486

Macintosh, B., Graham, J. R., Ingraham, P., et al. 2014, Proc. Natl. Acad. Sci. U.S.A., 111, 12661

Macintosh, B., Graham, J. R., Barman, T., et al. 2015, Science, 350, 64

Maire, A.-L., Langlois, M., Dohlen, K., et al. 2016, in Ground-based and Airborne Instrumentation for Astronomy VI, 9908, 990834

Mamajek, E. E., Bartlett, J. L., Seifahrt, A., et al. 2013, AJ, 146, 154

Marois, C., Lafrenière, D., Doyon, R., Macintosh, B., \& Nadeau, D. 2006, ApJ, 641, 556

Marois, C., Macintosh, B., Barman, T., et al. 2008, Science, 322, 1348

Marois, C., Zuckerman, B., Konopacky, Q. M., Macintosh, B., \& Barman, T. 2010, Nature, 468, 1080

Martinez, P., Dorrer, C., Aller Carpentier, E., et al. 2009, A\&A, 495, 363

Mawet, D., Milli, J., Wahhaj, Z., et al. 2014, ApJ, 792, 97

McElwain, M. W., \& Burgasser, A. J. 2006, AJ, 132, 2074

McLean, I. S., McGovern, M. R., Burgasser, A. J., et al. 2003, ApJ, 596, 561

McLean, I. S., Prato, L., McGovern, M. R., et al. 2007, ApJ, 658, 1217

Mellon, S. N., Mamajek, E. E., Oberst, T. E., \& Pecaut, M. J. 2017, ApJ, 844, 66

Mordasini, C., Alibert, Y., \& Benz, W. 2009a, A\&A, 501, 1139

Mordasini, C., Alibert, Y., Benz, W., \& Naef, D. 2009b, A\&A, 501, 1161

Mordasini, C., van Boekel, R., Mollière, P., Henning, T., \& Benneke, B. 2016, ApJ, 832, 41

Muench, A. A., Lada, C. J., Luhman, K. L., Muzerolle, J., \& Young, E. 2007, AJ, 134, 411

Mugrauer, M., Röll, T., Ginski, C., et al. 2012, MNRAS, 424, 1714

Nelder, J. A., \& Mead, R. 1965, Comput. J., 7, 308

Nielsen, E. L., De Rosa, R. J., Macintosh, B., et al. 2019, AJ, 158, 13

Öberg, K. I., Murray-Clay, R., \& Bergin, E. A. 2011, ApJ, 743, L16

Oliphant, T. E. 2006, A Guide to NumPy, Vol. 1 (Trelgol Publishing USA)

Ormel, C. W., \& Klahr, H. H. 2010, A\&A, 520, A43

Paardekooper, S. J., \& Mellema, G. 2004, A\&A, 425, L9

Pecaut, M. J., \& Mamajek, E. E. 2013, ApJS, 208, 9

Pecaut, M. J., \& Mamajek, E. E. 2016, MNRAS, 461, 794

Pedregosa, F., Varoquaux, G., Gramfort, A., et al. 2012, arXiv e-prints, [arXiv:1201.0490] 
A. J. Bohn et al.: Discovery of a directly imaged planet to the young solar analog YSES 2

Pereira, C. B., Franco, C. S., \& de Araújo, F. X. 2003, A\&A, 397, 927

Pollack, J. B., Hubickyj, O., Bodenheimer, P., et al. 1996, Icarus, 124, 62

Preibisch, T., \& Mamajek, E. 2008, The Nearest OB Association: ScorpiusCentaurus (Sco OB2), ASP Monograph Pub., 5, 235

Rafikov, R. R. 2005, ApJ, 621, L69

Rameau, J., Chauvin, G., Lagrange, A. M., et al. 2013, ApJ, 772, L15

Rayner, J. T., Cushing, M. C., \& Vacca, W. D. 2009, ApJS, 185, 289

Reid, I. N., Lewitus, E., Burgasser, A. J., \& Cruz, K. L. 2006, ApJ, 639, 1114

Schmidt, T. O. B., Neuhäuser, R., Seifahrt, A., et al. 2008, A\&A, 491, 311

Schwarz, H., Ginski, C., de Kok, R. J., et al. 2016, A\&A, 593, A74

Sheehan, P. 2020, Nature, 586, 205

Sheehan, P. D., Tobin, J. J., Federman, S., Megeath, S. T., \& Looney, L. W. 2020 ApJ, 902, 141

Sheppard, S. S., \& Cushing, M. C. 2009, AJ, 137, 304

Siegler, N., Close, L. M., Burgasser, A. J., et al. 2007, AJ, 133, 2320

Smith, B. A., \& Terrile, R. J. 1984, Science, 226, 1421

Soderblom, D. R., Jones, B. F., Balachandran, S., et al. 1993, AJ, 106, 1059

Soummer, R. 2005, ApJ, 618, L161

Soummer, R., Pueyo, L., \& Larkin, J. 2012, ApJ, 755, L28

Stolker, T., Bonse, M. J., Quanz, S. P., et al. 2019, A\&A, 621, A59

Stolker, T., Marleau, G. D., Cugno, G., et al. 2020a, A\&A, 644, A13
Stolker, T., Quanz, S. P., Todorov, K. O., et al. 2020b, A\&A, 635, A182 Torres, C. A. O., Quast, G. R., da Silva, L., et al. 2006, A\&A, 460, 695

Van der Walt, S., Schönberger, J. L., Nunez-Iglesias, J., et al. 2014, PeerJ, 2, e453 van Terwisga, S. E., van Dishoeck, E. F., Ansdell, M., et al. 2018, A\&A, 616, A88

Vigan, A., Patience, J., Marois, C., et al. 2012, A\&A, 544, A9

Vigan, A., Bonavita, M., Biller, B., et al. 2017, A\&A, 603, A3

Vigan, A., Fontanive, C., Meyer, M., et al. 2021, A\&A, in press, https://doi.org/10.1051/0004-6361/202038107

Virtanen, P., Gommers, R., Oliphant, T. E., et al. 2020, Nat. Methods, 17, 261

Voges, W., Aschenbach, B., Boller, T., et al. 1999, A\&A, 349, 389

Wahhaj, Z., Milli, J., Romero, C., et al. 2021, A\&A, 648, A26

Wang, J. J., Graham, J. R., Dawson, R., et al. 2018, AJ, 156, 192

Wang, J. J., Ginzburg, S., Ren, B., et al. 2020, AJ, 159, 263

Wang, J. J., Vigan, A., Lacour, S., et al. 2021, AJ, 161, 148

Wenger, M., Ochsenbein, F., Egret, D., et al. 2000, A\&AS, 143, 9

Wertz, O., Absil, O., Gómez González, C. A., et al. 2017, A\&A, 598, A83

Zari, E., Hashemi, H., Brown, A. G. A., Jardine, K., \& de Zeeuw, P. T. 2018 , A\&A, 620, A172

Zhu, Z., Hartmann, L., Nelson, R. P., \& Gammie, C. F. 2012, ApJ, 746, 110

Zuckerman, B., Rhee, J. H., Song, I., \& Bessell, M. S. 2011, ApJ, 732, 61 


\section{Appendix A: Observational conditions and setup}

We present the observational setup and the weather conditions for our SPHERE observations in Table A.1.

Table A.1. SPHERE observations of YSES 2.

\begin{tabular}{llllllll}
\hline \hline $\begin{array}{l}\text { Observation date } \\
\text { (yyyy-mm-dd) }\end{array}$ & Filter & $\begin{array}{l}F W H M^{(a)} \\
(\mathrm{mas})\end{array}$ & $\begin{array}{l}\text { NEXP } \times \text { NDIT } \times \mathrm{DIT}^{(b)} \\
(1 \times 1 \times \mathrm{s})\end{array}$ & $\begin{array}{l}\Delta \pi^{(c)} \\
\left(^{\circ}\right)\end{array}$ & $\begin{array}{l}\langle\omega\rangle^{(d)} \\
\left({ }^{\prime \prime}\right)\end{array}$ & $\langle X\rangle^{(e)}$ & $\begin{array}{l}\left\langle\tau_{0}\right\rangle^{(f)} \\
(\mathrm{ms})\end{array}$ \\
\hline $2018-04-30$ & $H$ & 50.5 & $\begin{array}{l}4 \times 1 \times 32 \\
1 \times 18 \times 16\end{array}$ & 0.98 & 0.87 & 1.343 & 6.25 \\
$2020-12-08$ & $K_{\mathrm{s}}$ & 61.7 & 1.37 & 0.55 & 1.52 & 4.30 \\
\hline
\end{tabular}

Notes. ${ }^{(a)}$ Full width at half maximum measured for the non-coronagraphic stellar PSF. ${ }^{(b)}$ NEXP describes the number of exposures, NDIT is the number of subintegrations per exposure, and DIT is the detector integration time of an individual subintegration. ${ }^{(c)} \Delta \pi$ describes the amount of field rotation during the observation, if it is carried out in pupil-stabilized mode (only valid for CI observations). ${ }^{(d)}\langle X\rangle$ denotes the average airmass during the observation. ${ }^{(e)}\langle\omega\rangle$ denotes the average seeing conditions during the observation. ${ }^{(f)}\left\langle\tau_{0}\right\rangle$ denotes the average coherence time during the observation.

\section{Appendix B: Reference library}

The reference libraries were compiled from the full amount of YSES data that were collected under ESO IDs 099.C0698(A) (PI: Kenworthy), 0101.C-0153(A) (PI: Kenworthy), 0101.C-0341(A) (PI: Bohn), and 106.20X2.001 (PI: Vogt). We used RDI in the innermost region of the images $<1$.' 2 , where the stellar halo was dominating the received flux. We deselected all targets with obvious point sources or extended structures in this region because these signals are not part of the stellar PSF and would therefore deteriorate the quality of our model created by PCA. The remaining targets, their observation epochs, and observing conditions that were used as a reference library for the $H$ and $K_{\mathrm{s}}$ band data are listed in Tables B.1 and B.2, respectively. In the $H$ band, we have 269 individual reference frames and in the $K_{\mathrm{s}}$ band we have 164 . 
Table B.1. Reference library for the data reduction in $H$ band.

\begin{tabular}{|c|c|c|c|c|c|}
\hline $\begin{array}{l}\text { Target } \\
\text { (2MASS ID) }\end{array}$ & $\begin{array}{l}\text { Observation date } \\
\text { (yyyy-mm-dd) }\end{array}$ & $\begin{array}{l}\mathrm{NEXP} \times \mathrm{NDIT} \times \mathrm{DIT}^{(a)} \\
(1 \times 1 \times \mathrm{s})\end{array}$ & $\begin{array}{l}\langle\omega\rangle^{(b)} \\
\left({ }^{\prime \prime}\right)\end{array}$ & $\langle X\rangle^{(c)}$ & $\begin{array}{l}\left\langle\tau_{0}\right\rangle^{(d)} \\
(\mathrm{ms})\end{array}$ \\
\hline J11272881-3952572 & 2017-04-18 & $4 \times 1 \times 32$ & 1.51 & 1.10 & 1.40 \\
\hline J11320835-5803199 & 2017-06-17 & $4 \times 1 \times 32$ & 0.67 & 1.47 & 2.90 \\
\hline J11445217-6438548 & 2018-05-14 & $4 \times 1 \times 32$ & 0.72 & 1.31 & 2.38 \\
\hline J11454278-5739285 & $2018-06-04$ & $4 \times 1 \times 32$ & 0.70 & 1.19 & 2.80 \\
\hline J11454278-5739285 & 2019-01-13 & $4 \times 1 \times 32$ & 1.14 & 1.62 & 3.83 \\
\hline J12065276-5044463 & 2017-04-02 & $3 \times 1 \times 32$ & 1.24 & 1.11 & 1.50 \\
\hline J12090225-5120410 & $2018-05-15$ & $4 \times 1 \times 32$ & 0.86 & 1.12 & 2.70 \\
\hline J12090225-5120410 & 2019-12-14 & $12 \times 2 \times 32$ & 0.63 & 1.51 & 7.75 \\
\hline J12101065-4855476 & 2017-04-18 & $4 \times 1 \times 32$ & 1.71 & 1.15 & 1.40 \\
\hline J12113142-5816533 & $2018-12-22$ & $3 \times 2 \times 32$ & 1.46 & 1.47 & 2.13 \\
\hline J12113142-5816533 & 2019-02-18 & $4 \times 2 \times 32$ & 0.45 & 1.23 & 14.30 \\
\hline J12160114-5614068 & $2018-12-27$ & $4 \times 2 \times 32$ & 0.41 & 1.45 & 11.88 \\
\hline J12164023-7007361 & $2018-12-23$ & $3 \times 1 \times 32$ & 0.98 & 1.59 & 2.93 \\
\hline J12164023-7007361 & 2019-02-15 & $4 \times 1 \times 32$ & 0.54 & 1.63 & 11.20 \\
\hline J12185802-5737191 & 2017-06-17 & $2 \times 1 \times 32$ & 0.72 & 1.22 & 2.70 \\
\hline J12195938-5018404 & $2018-12-30$ & $4 \times 1 \times 32$ & 0.53 & 1.62 & 8.00 \\
\hline J12210499-7116493 & 2019-01-12 & $4 \times 2 \times 32$ & 0.80 & 1.53 & 4.25 \\
\hline $\mathrm{J} 12220430-4841248$ & $2017-04-18$ & $3 \times 1 \times 32$ & 1.82 & 1.17 & 1.40 \\
\hline $\mathrm{J} 12234012-5616325$ & 2017-06-17 & $4 \times 1 \times 32$ & 0.62 & 1.72 & 3.45 \\
\hline J12264842-5215070 & $2018-12-30$ & $4 \times 1 \times 32$ & 0.40 & 1.38 & 8.20 \\
\hline $\mathrm{J} 12302957-5222269$ & $2018-12-30$ & $4 \times 1 \times 32$ & 0.38 & 1.33 & 9.85 \\
\hline J12333381-5714066 & 2019-01-01 & $4 \times 1 \times 32$ & 0.76 & 1.37 & 7.03 \\
\hline J12333381-5714066 & 2019-01-14 & $4 \times 1 \times 32$ & 1.26 & 1.21 & 2.45 \\
\hline J12361767-5042421 & $2018-12-30$ & $4 \times 1 \times 32$ & 0.51 & 1.59 & 4.68 \\
\hline J12361767-5042421 & $2019-12-18$ & $16 \times 2 \times 32$ & 1.14 & 1.57 & 3.07 \\
\hline J12374883-5209463 & $2018-12-30$ & $4 \times 1 \times 32$ & 0.41 & 1.50 & 7.30 \\
\hline J12383556-5916438 & 2019-01-03 & $4 \times 1 \times 32$ & 0.52 & 1.59 & 13.90 \\
\hline J12383556-5916438 & 2019-01-12 & $4 \times 1 \times 32$ & 0.79 & 1.26 & 4.25 \\
\hline J12393796-5731406 & 2017-06-17 & $4 \times 1 \times 32$ & 0.64 & 1.77 & 3.83 \\
\hline J12404664-5211046 & 2018-04-30 & $4 \times 1 \times 32$ & 0.74 & 1.13 & 7.05 \\
\hline J12442412-5855216 & 2017-06-17 & $4 \times 3 \times 32$ & 0.71 & 1.37 & 2.67 \\
\hline J12454884-5410583 & 2018-04-30 & $4 \times 1 \times 32$ & 0.71 & 1.15 & 6.92 \\
\hline J12480778-4439167 & 2017-06-17 & $4 \times 2 \times 32$ & 0.90 & 1.34 & 2.75 \\
\hline J12505143-5156353 & 2019-01-12 & $4 \times 1 \times 32$ & 1.14 & 1.32 & 3.75 \\
\hline J12510556-5253121 & 2019-01-08 & $4 \times 1 \times 32$ & 0.58 & 1.68 & 3.90 \\
\hline J13015069-5304581 & 2019-01-08 & $4 \times 1 \times 32$ & 0.55 & 1.60 & 3.95 \\
\hline J13055087-5304181 & 2018-06-11 & $4 \times 1 \times 32$ & 0.82 & 1.14 & 1.95 \\
\hline J13055087-5304181 & 2018-07-04 & $4 \times 1 \times 32$ & 1.73 & 1.14 & 1.70 \\
\hline J13064012-5159386 & $2018-04-30$ & $4 \times 1 \times 32$ & 0.56 & 1.13 & 8.15 \\
\hline J13065439-4541313 & 2018-04-08 & $4 \times 1 \times 32$ & 0.46 & 1.09 & 5.65 \\
\hline J13095880-4527388 & 2018-05-01 & $4 \times 1 \times 32$ & 1.08 & 1.07 & 2.70 \\
\hline J13103245-4817036 & 2018-05-01 & $4 \times 1 \times 32$ & 1.03 & 1.09 & 3.30 \\
\hline J13121764-5508258 & $2017-08-31$ & $4 \times 1 \times 32$ & 0.68 & 2.22 & 4.42 \\
\hline J13121764-5508258 & $2018-05-15$ & $4 \times 1 \times 32$ & 0.62 & 1.16 & 2.50 \\
\hline J13174687-4456534 & $2018-05-28$ & $4 \times 1 \times 32$ & 0.70 & 1.07 & 4.33 \\
\hline J13334410-6359345 & 2017-07-05 & $4 \times 1 \times 32$ & 1.06 & 1.53 & 3.05 \\
\hline J13343188-4209305 & 2017-04-02 & $4 \times 1 \times 32$ & 1.14 & 1.21 & 1.70 \\
\hline J13354082-4818124 & 2017-04-02 & $4 \times 1 \times 32$ & 1.06 & 1.30 & 2.08 \\
\hline J13380596-4344564 & 2017-04-02 & $4 \times 1 \times 32$ & 1.05 & 1.32 & 2.40 \\
\hline J13455599-5222255 & $2018-04-28$ & $4 \times 1 \times 32$ & 0.64 & 1.13 & 6.35 \\
\hline
\end{tabular}

Notes. ${ }^{(a)}$ NEXP describes the number of exposures, NDIT is the number of subintegrations per exposure, and DIT is the detector integration time of an individual subintegration. ${ }^{(b)}\langle X\rangle$ denotes the average airmass during the observation. ${ }^{(c)}\langle\omega\rangle$ denotes the average seeing conditions during the observation. ${ }^{(d)}\left\langle\tau_{0}\right\rangle$ denotes the average coherence time during the observation. 
Table B.2. Reference library for the data reduction in $K_{\mathrm{s}}$ band.

\begin{tabular}{|c|c|c|c|c|c|}
\hline $\begin{array}{l}\text { Target } \\
\text { (2MASS ID) }\end{array}$ & $\begin{array}{l}\text { Observation date } \\
\text { (yyyy-mm-dd) }\end{array}$ & $\begin{array}{l}\mathrm{NEXP} \times \mathrm{NDIT} \times \mathrm{DIT}^{(a)} \\
(1 \times 1 \times \mathrm{s})\end{array}$ & $\begin{array}{l}\langle\omega\rangle^{(b)} \\
\left({ }^{\prime \prime}\right)\end{array}$ & $\langle X\rangle^{(c)}$ & $\begin{array}{l}\left\langle\tau_{0}\right\rangle^{(d)} \\
(\mathrm{ms})\end{array}$ \\
\hline J11445217-6438548 & 2018-05-14 & $4 \times 1 \times 32$ & 0.77 & 1.31 & 2.60 \\
\hline J11454278-5739285 & 2019-01-13 & $4 \times 1 \times 32$ & 1.18 & 1.59 & 3.58 \\
\hline J12090225-5120410 & $2018-05-15$ & $4 \times 1 \times 32$ & 0.70 & 1.12 & 2.90 \\
\hline J12113142-5816533 & $2018-12-22$ & $4 \times 2 \times 32$ & 1.38 & 1.44 & 2.05 \\
\hline J12113142-5816533 & 2019-02-18 & $4 \times 2 \times 32$ & 0.45 & 1.22 & 15.00 \\
\hline J12160114-5614068 & $2018-12-27$ & $4 \times 2 \times 32$ & 0.47 & 1.42 & 10.27 \\
\hline J12164023-7007361 & $2018-12-23$ & $4 \times 1 \times 32$ & 1.06 & 1.58 & 3.43 \\
\hline J12164023-7007361 & $2019-02-15$ & $4 \times 1 \times 32$ & 0.57 & 1.61 & 10.75 \\
\hline J12195938-5018404 & $2018-12-30$ & $4 \times 1 \times 32$ & 0.55 & 1.59 & 9.00 \\
\hline J12210499-7116493 & 2019-01-12 & $4 \times 2 \times 32$ & 0.82 & 1.52 & 4.40 \\
\hline J12264842-5215070 & $2018-12-30$ & $4 \times 1 \times 32$ & 0.41 & 1.36 & 9.20 \\
\hline $\mathrm{J} 12302957-5222269$ & $2018-12-30$ & $4 \times 1 \times 32$ & 0.45 & 1.32 & 7.48 \\
\hline J12333381-5714066 & 2019-01-01 & $4 \times 1 \times 32$ & 0.80 & 1.36 & 6.25 \\
\hline J12333381-5714066 & 2019-01-14 & $4 \times 1 \times 32$ & 1.24 & 1.21 & 2.30 \\
\hline J12333381-5714066 & $2020-12-10$ & $1 \times 20 \times 16$ & 0.58 & 1.76 & 5.50 \\
\hline J12361767-5042421 & $2018-12-30$ & $4 \times 1 \times 32$ & 0.47 & 1.56 & 6.22 \\
\hline J12374883-5209463 & $2018-12-30$ & $4 \times 1 \times 32$ & 0.46 & 1.48 & 6.95 \\
\hline J12383556-5916438 & 2019-01-03 & $4 \times 1 \times 32$ & 0.46 & 1.56 & 12.47 \\
\hline J12383556-5916438 & 2019-01-12 & $4 \times 1 \times 32$ & 0.94 & 1.26 & 3.45 \\
\hline J12404664-5211046 & 2018-04-30 & $4 \times 1 \times 32$ & 0.87 & 1.13 & 7.10 \\
\hline J12454884-5410583 & $2018-04-30$ & $4 \times 1 \times 32$ & 0.66 & 1.15 & 8.97 \\
\hline J12505143-5156353 & 2019-01-12 & $4 \times 1 \times 32$ & 1.03 & 1.31 & 4.10 \\
\hline J12510556-5253121 & 2019-01-08 & $4 \times 1 \times 32$ & 0.52 & 1.65 & 3.98 \\
\hline J13015069-5304581 & 2019-01-08 & $4 \times 1 \times 32$ & 0.49 & 1.58 & 4.80 \\
\hline J13055087-5304181 & 2018-06-11 & $4 \times 1 \times 32$ & 0.93 & 1.14 & 2.02 \\
\hline J13055087-5304181 & 2018-07-04 & $4 \times 1 \times 32$ & 1.73 & 1.14 & 1.70 \\
\hline J13064012-5159386 & $2018-04-30$ & $4 \times 1 \times 32$ & 0.56 & 1.13 & 9.88 \\
\hline J13065439-4541313 & 2018-04-08 & $4 \times 1 \times 32$ & 0.55 & 1.09 & 4.68 \\
\hline J13095880-4527388 & 2018-05-01 & $4 \times 1 \times 32$ & 1.03 & 1.07 & 2.45 \\
\hline $\mathrm{J} 13103245-4817036$ & $2018-05-01$ & $4 \times 1 \times 32$ & 0.87 & 1.10 & 4.40 \\
\hline J13121764-5508258 & $2018-05-15$ & $4 \times 1 \times 32$ & 0.62 & 1.16 & 3.00 \\
\hline J13174687-4456534 & $2018-05-28$ & $4 \times 1 \times 32$ & 0.67 & 1.07 & 4.15 \\
\hline J13455599-5222255 & 2018-04-28 & $4 \times 1 \times 32$ & 0.65 & 1.13 & 6.03 \\
\hline
\end{tabular}

Notes. ${ }^{(a)}$ NEXP describes the number of exposures, NDIT is the number of subintegrations per exposure, and DIT is the detector integration time of an individual subintegration. ${ }^{(b)}\langle X\rangle$ denotes the average airmass during the observation. ${ }^{(c)}\langle\omega\rangle$ denotes the average seeing conditions during the observation. ${ }^{(d)}\left\langle\tau_{0}\right\rangle$ denotes the average coherence time during the observation. 


\section{Appendix C: Extraction of companion astrometry and photometry}

We extracted the companion astrometry and photometry with the SimplexMinimizationModule of PynPoint. This injects an artificial planet into the data prior to the stellar PSF subtraction with RDI plus PCA. The planet template PSF is obtained from the unsaturated, non-coronagraphic flux images that were taken alongside the observations. The methods injects the artificial planet into the data at the approximate position and magnitude of the real point source, considering the parallactic rotation during the observing sequence. The PSF subtraction is performed using the same library as before (see Appendix B), we smooth the image with a Gaussian kernel with a FWHM of 12 mas (which corresponds to the size of a detector pixel) to reduce pixel-topixel variations, and we evaluate the residuals in an aperture with a diameter of $\sim 0$.' 25 around the injection position. We choose the image curvature, which is represented by the determinant of the Hessian matrix as function of merit, which we aim to minimize by varying the input separation, position angle, and magnitude contrast of our artificial companion. We do not use the absolute value norm as presented by Wertz et al. (2017) as an objective to the minimization because this would not consider large-scale features in the residual image that are not correctly modeled by our PSF subtraction approach. Such a feature is for instance the asymmetric wind driven halo (Cantalloube et al. 2018) that is apparent in the $K_{\mathrm{s}}$ band data in the northeastern to southwestern direction (see right panel of Fig. 2). This uncorrected stellar flux contributes to the planet signal and minimization of the absolute value norm around the planet position would certainly overestimate its flux and perhaps even compromise its astrometry. Planet separation, position angle, and magnitude contrast are optimized simultaneously by a Nelder-Mead simplex minimization algorithm (Nelder \& Mead 1965).

Owing to the optimization process our final values for the planetary astrometry and photometry do not exhibit any intrinsic uncertainties. To derive the systematic uncertainties of our injection and minimization approach, we follow the analysis described by Stolker et al. (2020b), using the cube in which the optimized negative planet is injected such that no companion signal remains in the data. For 24 position angles that are equidistantly distributed in polar space we inject positive artificial companions into the data using the same magnitude contrast and the same radial separation as previously determined for our companion. We extract the astrometry and photometry of these artificial companions with the same method as described before and we evaluate the deviations from the injection position and flux. The standard deviation along the 24 distinct positions is utilized as uncertainty of our extraction method. These are combined with additional astrometric uncertainties originating from the detector plate scale, the true north offset, and the centering accuracy of 2.5 mas (see SPHERE manual) to derive the final value of planet separation and position angle as presented in Table 2. For the companion photometry, we add uncertainties due to the variation of the unsaturated stellar PSF throughout the sequence of flux measurements and we account for transmissivity variations of the neutral density filter across the broadband filter that was used for our observations (either the $H$ or $K_{\mathrm{s}}$ band).

\section{Appendix D: Astrometric analysis of background objects}

In addition to YSES 2b, there are four candidate companions (CCs) in the SPHERE/IRDIS field of view that we could identify in both observational epochs. These CCs are presented in Fig. D.1, in which we show the de-rotated data from the night of 2020 December 12. No PSF subtraction with RDI is performed, instead we just applied an unsharp mask with a Gaussian kernel size of 5 pixels. YSES $2 b$ can easily be identified in this image product as well. For the remaining CCs, we present the relative astrometric offsets between both observational epochs in the proper motion diagram in Fig. D.2. The relative motions of CCs 2-5 are clearly compatible with stationary background objects, and co-movement can be ruled out for all of them.

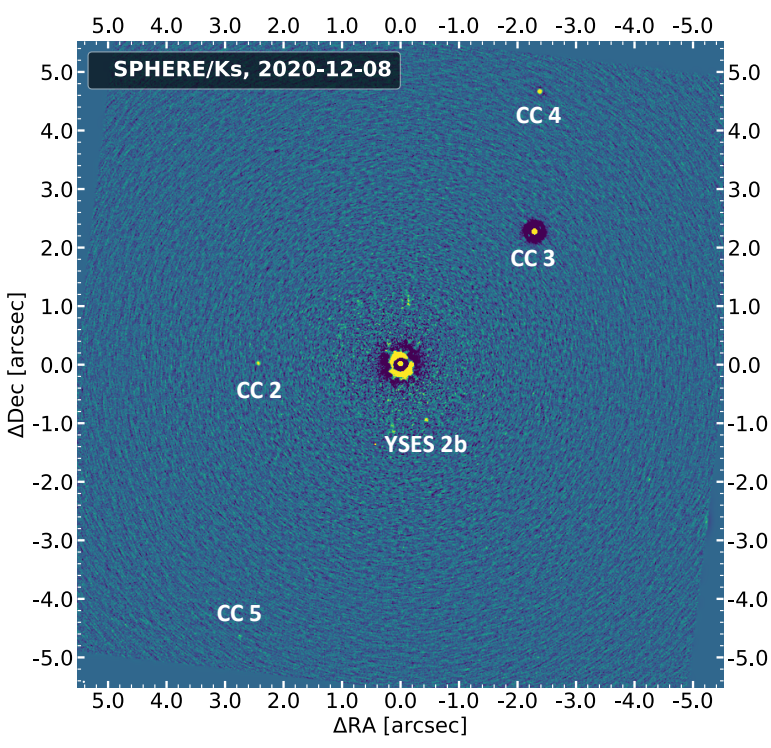

Fig. D.1. Reduced SPHERE data for the full IRDIS field of view. The images are de-rotated and median combined; an unsharp mask is applied to remove the stellar halo. Four additional companion candidates to YSES $2 b$ are identified in the field of view. The image is presented at an arbitrary logarithmic color scale to highlight the off-axis point sources.

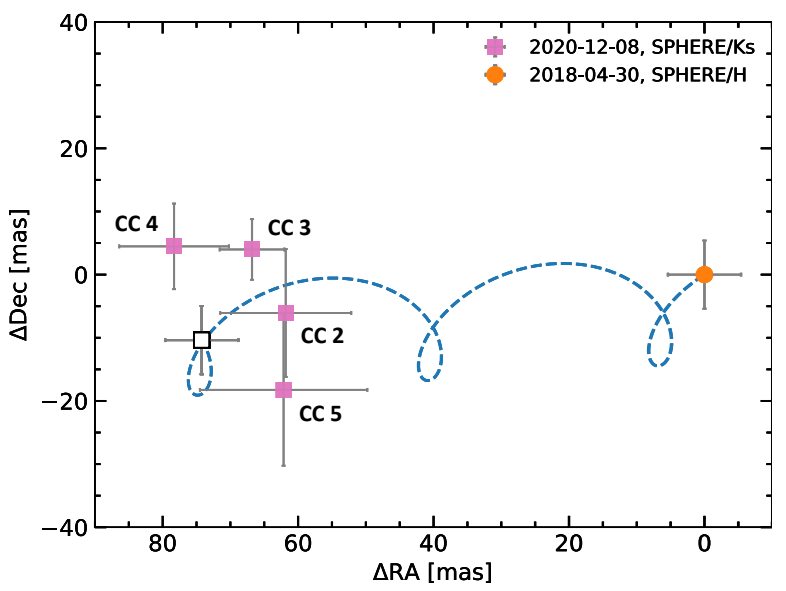

Fig. D.2. Proper motion plot for background objects in the SPHERE/IRDIS field of view. The pink markers indicate the relative astrometric offsets to the first observational epoch that is plotted at the origin of the coordinate system (orange marker). The blue trajectory represents the simulated motion of a static background object at infinity and the white marker shows the relative positional offset of such an object at the time of our second observation. 